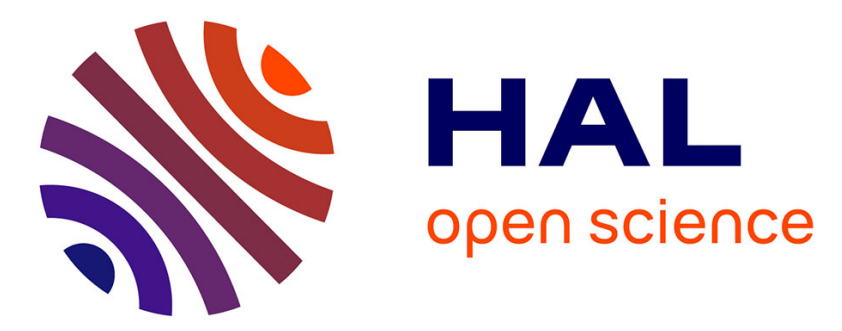

\title{
Hydrologie et environnement à la périphérie de Nîmes (Gard) : le paléochenal de la ZAC de la gare.
}

Odile Maufras, Pascale Chevillot, Dominique Peyric, Jacqueline Argant, Sébastien Barberan, Farid Sellami

\section{To cite this version:}

Odile Maufras, Pascale Chevillot, Dominique Peyric, Jacqueline Argant, Sébastien Barberan, et al.. Hydrologie et environnement à la périphérie de Nîmes (Gard): le paléochenal de la ZAC de la gare.. Revue archéologique de Narbonnaise, 2003, 36 (1), pp.25-42. 10.3406/ran.2003.1116 . halshs01739876

\section{HAL Id: halshs-01739876 \\ https://shs.hal.science/halshs-01739876}

Submitted on 9 Feb 2021

HAL is a multi-disciplinary open access archive for the deposit and dissemination of scientific research documents, whether they are published or not. The documents may come from teaching and research institutions in France or abroad, or from public or private research centers.
L'archive ouverte pluridisciplinaire HAL, est destinée au dépôt et à la diffusion de documents scientifiques de niveau recherche, publiés ou non, émanant des établissements d'enseignement et de recherche français ou étrangers, des laboratoires publics ou privés. 


\section{Hydrologie et environnement à la périphérie de Nîmes (Gard) : le} paléochenal de la ZAC de la gare

Odile Maufras, Pascale Chevillot, Dominique Peyric, Jacqueline Argant, Sébastien Barberan, Farid Sellami

\section{Citer ce document / Cite this document :}

Maufras Odile, Chevillot Pascale, Peyric Dominique, Argant Jacqueline, Barberan Sébastien, Sellami Farid. Hydrologie et environnement à la périphérie de Nîmes (Gard) : le paléochenal de la ZAC de la gare. In: Revue archéologique de Narbonnaise, tome 36, 2003. pp. 25-42;

doi : https://doi.org/10.3406/ran.2003.1116

https://www.persee.fr/doc/ran_0557-7705_2003_num_36_1_1116

Fichier pdf généré le 27/10/2018 


\title{
Résumé
}

La fouille d'un paléochenal au sud de Nîmes a touché un bord et une large partie des remplissages de l'ancien thalweg; ces derniers ont fait l'objet d'analyses archéologiques et paléoenvironnementales qui ont révélé trois modes de fonctionnement successifs du cours d'eau. Jusqu'au ler $\mathbf{s}$. de n. è., il est dynamique et bordé d'un paysage ouvert, cultivé. Ensuite et jusqu'à la fin du lle s. son activité diminue au point qu'un chemin est installé dans son lit tandis que le paysage alentour se referme. Enfin, à partir du Ille s., l'activité alluviale cesse et l'érosion des versants entraîne le colmatage du lit qui disparaît du paysage au plus tard au XIXe s. Cette évolution s'explique par le double impact du climat et de l'activité des hommes, particulièrement l'urbanisation du site de Nîmes au ler $\mathbf{s}$.

\begin{abstract}
The excavation of a paleovalley southwards of Nîmes concerned a side and a great part of the fillings of an ancient talweg : these fillings have been analysed on behalf of archaeology and paleoenvironment. These analysises revealed three succeding ways of proceeding of the stream. It is dynamic and an open cultivated landscape lines it until the Isl century A.D. Then and until the end of the IInd century A.D., the stream activity is so diminished that a path is made in the stream bed while the landscape enclosed. Then and until the end of the IIIrd century A.D., the alluvial activity stops and the erosion of the sides leads to the warping of the stream bed disappeared from the landscape more lately during the XIXth century. Explanation of this evolution is in the double impact of climate and human activities, especially the urbanization of the site of Nîmes during the first century A.D.
\end{abstract}




\title{
Hydrologie et environnement à la périphérie de Nîmes (Gard) : le paléochenal de la ZAC de la gare
}

\author{
Odile MAUFRAS *, Pascale CHEVILLOT * et Dominique PEYRIC ** \\ avec les contributions de Jacqueline ARGANT ***, Sébastien BARBERAN * et Farid SELLAMI *
}

\begin{abstract}
Résumé : La fouille d'un paléochenal au sud de Nîmes a touché un bord et une large partie des remplissages de l'ancien thalweg; ces derniers ont fait l'objet d'analyses archéologiques et paléoenvironnementales qui ont révélé trois modes de fonctionnement successifs du cours d'eau. Jusqu'au $\mathrm{I}^{\mathrm{er}} \mathrm{s}$. de n. è., il est dynamique et bordé d'un paysage ouvert, cultivé. Ensuite et jusqu'à la fin du $\mathrm{II}^{\mathrm{e}} \mathrm{s}$. son activité diminue au point qu'un chemin est installé dans son lit tandis que le paysage alentour se referme. Enfin, à partir du III $^{e}$ s., l'activité alluviale cesse et l'érosion des versants entraîne le colmatage du lit qui disparaît du paysage au plus tard au XIX ${ }^{\mathrm{e}} \mathrm{s}$. Cette évolution s'explique par le double impact du climat et de l'activité des hommes, Ğarticulièrement l'urbanisation du site de Nîmes au Ier $s$.

Mots-clés : Nîmes, paléo-environnement, hydrologie, voirie, Antiquité.

Abstract : The excavation of a paleovalley southwards of Nîmes concerned a side and a great part of the fillings of an ancient talweg : these $t_{1}$ 'lings have been analysed on behalf of archaeology and paleoenvironment. These analysises revealed three succeding ways of proceeding of the siream. It is dynamic and an open cultivated landscape lines it until the $1^{\text {st }}$ century A.D. Then and until the end of the II ${ }^{\text {nd }}$ century A.D., the stream ativity is so diminished that a path is made in the stream bed while the landscape enclosed. Then and until the end of the III ${ }^{\text {rd }}$ century A.D., the alluval activity stops and the erosion of the sides leads to the warping of the stream bed disappeared from the landscape more lately during the XIX ${ }^{\text {th }}$ centh ry. Explanation of this evolution is in the double impact of climate and human activities, especially the urbanization of the site of Nîmes during the fist century A.D.
\end{abstract}

Keywords : Nîmes, paleoenvironment, hydrology, ways, Antiquity.

\section{Introduction}

Les travaux de réaménagement du quartier de la gare de Nîmes ont donné lieu, au printemps 2000 , à une série de petites opérations archéologiques qui ont révélé, outre quelques traces de culture antique (fosses et fossés), la présence d'un ancien chenal '. Ce dernier participe du réseau des vallons qui drainent les eaux de la garrigue et du piémont, au nord-ouest de la ville, vers la plaine du Vistre, au sud-est (fig. 1). Il a été progressivement colmaté au cours de la période historique et ainsi il constitue un témoin des fluctuations hydro-sédimentaires de notre ère, de l'évolution de l'environnement que celles-ci reflètent et, en corollaire, de l'impact de l'occupation anthropique.
Cet article rend compte des résultats de la brève fouille de sauvetage menée sur le tronçon accessible de l'ancien cours d'eau et des analyses paléoenvironnementales des échantillons prélevés dans ses différents remplissages ${ }^{2}$. L'intervention archéologique a permis la reconnaissance de modes de fonctionnement successifs du vallon entre le $\mathrm{I}^{\mathrm{er}} \mathrm{s}$. av. n.è. et le XIX ${ }^{\mathrm{e}} \mathrm{s}$., modes auxquels sont associées une évolution de l'occupation et une transformation progressive de l'environnement. Jusqu'au milieu ou la fin du $\mathrm{II}^{\mathrm{e}} \mathrm{s}$. de n.è., le chenal a fonctionné en cours d'eau actif dans un paysage relativement humide et encore un peu boisé. L'assèchement progressif du cours d'eau a permis l'installation d'une voie de circulation dans son lit et par la suite son comblement progressif par des apports collu-

1 Les opérations archéologiques sont les suivantes: "ZAC de la gare I-2000", diagnostic archéologique sous la direction de Yves Manniez (Inrap); "ZAC de la gare II -2001", fouille préventive du paléochenal de la gare sous la direction de Odile Maufras (Inrap); "ZAC de la gare III -2000", diagnostic archéologique sous la direction de Odile Maufras.

2 L'opération a été menée sur le terrain en mai 2000. Elle a duré 2 semaines et a été réalisée par une équipe de trois archéologues et un géomorphologue. Les analyses des échantillons et l'étude des résultats se sont échelonnées jusqu’en septembre 2001. 


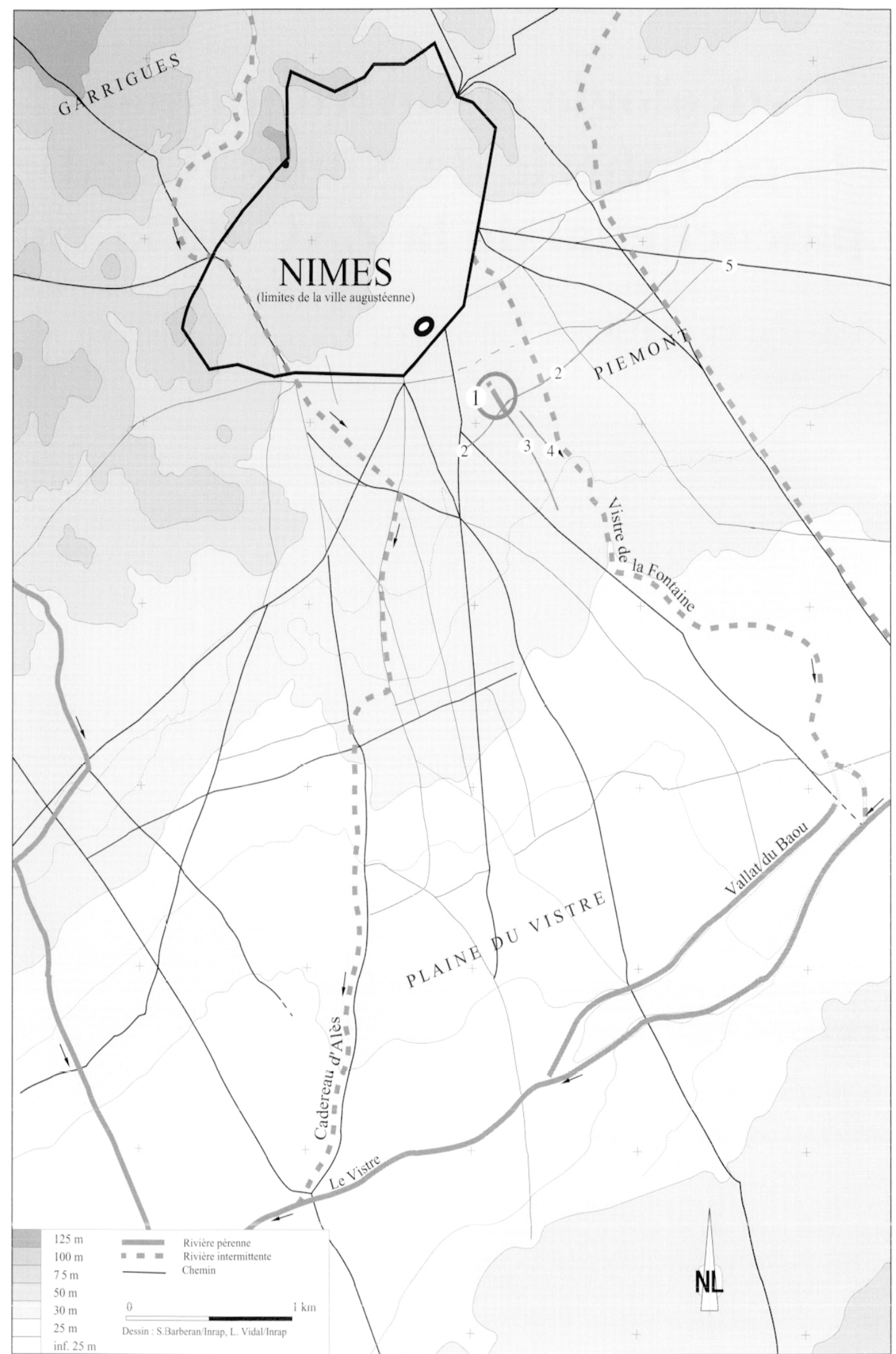

Fig. I. Localisation du site. I: paléochenal de la gare. 2 : rues Sainte-Félicité et Sainte-Perpétue. 3: rue des Marronniers. 4: rue Quatrefages. 5 : tracé de la voie domitienne (dessin IGN et G. Barberan, L. Vidal, Inrap). 
viaux. Ces derniers se mettent en place jusqu'à l'époque moderne en plusieurs phases d'accumulation, séparées par des périodes de stabilité qui ont permis le développement de sols. La culture des terres voisines est attestée par la découverte de fosses de plantation du I ${ }^{\text {er }}$ s. av. n.è. et du Haut Empire; elle s'intensifie sans doute par la suite pour aboutir à la plus grande ouverture du paysage observée dans les niveaux supérieurs, supposés antiques tardifs, médiévaux puis modernes.

Les apports de la fouille du paléochenal de la gare II forment un lot de données importantes pour préciser la connaissance de l'environnement en plaine du Vistre et aux abords de la ville de Nîmes. Ces données sont néanmoins encore partielles et il conviendra de les exploiter dans un cadre plus large, notamment en les confrontant aux apports des travaux archéologiques menés en amont du plan de protection contre les inondations et du programme collectif de recherches sur l'espace rural de la région nîmoise. Cependant, elles apportent d'ores et déjà un éclairage sur les incidences de l'évolution du milieu et de l'occupation humaine, au premier chef le développement de l'urbanisme au début de notre ère, sur la transformation du paysage.

\section{Présentation du contexte nîmois}

\subsection{Le cadre géographique}

Le site de Nîmes s'appuie au nord sur le massif de collines et de plateaux calcaires de la Garrigue. Les reliefs modérés de celle-ci s'étagent pour former un vaste amphithéâtre qui s'ouvre au sud sur le piémont et protège la ville des vents dominants (Fabre 2000, 22; Monteil 1999, 19). L'agglomération est implantée en amont du piémont, sur une pente qui descend doucement au sud vers la plaine du Vistre, dépression humide incisée selon une orientation est-ouest (fig. 1).

La Garrigue est entaillée par des ruisseaux qui lui sont perpendiculaires (nord-sud) et qui ont drainé ses eaux vers la plaine alluviale. Ces talwegs, pour la plupart, se sont progressivement colmatés au point de disparaitre du paysage, en aval. L'écoulement des eaux s'est alors trouvé relayé par les aquifères souterrains (Fabre 2000, 26). Néanmoins deux subsistent: le Vistre de la Fontaine qui a été partiellement canalisé à l'époque moderne et le cadereau d'Alès ${ }^{3}$ (fig. 2). Ces cours fonctionnent actuellement de manière intermittente: ils restent la plupart du temps à sec et ne se remplissent que lors des orages violents, selon un processus classique en domaine climatique méditerranéen (Chevillot 1999, 40).

Le paléochenal découvert dans le quartier de la gare s'inscrit dans ce système hydrologique de surface.

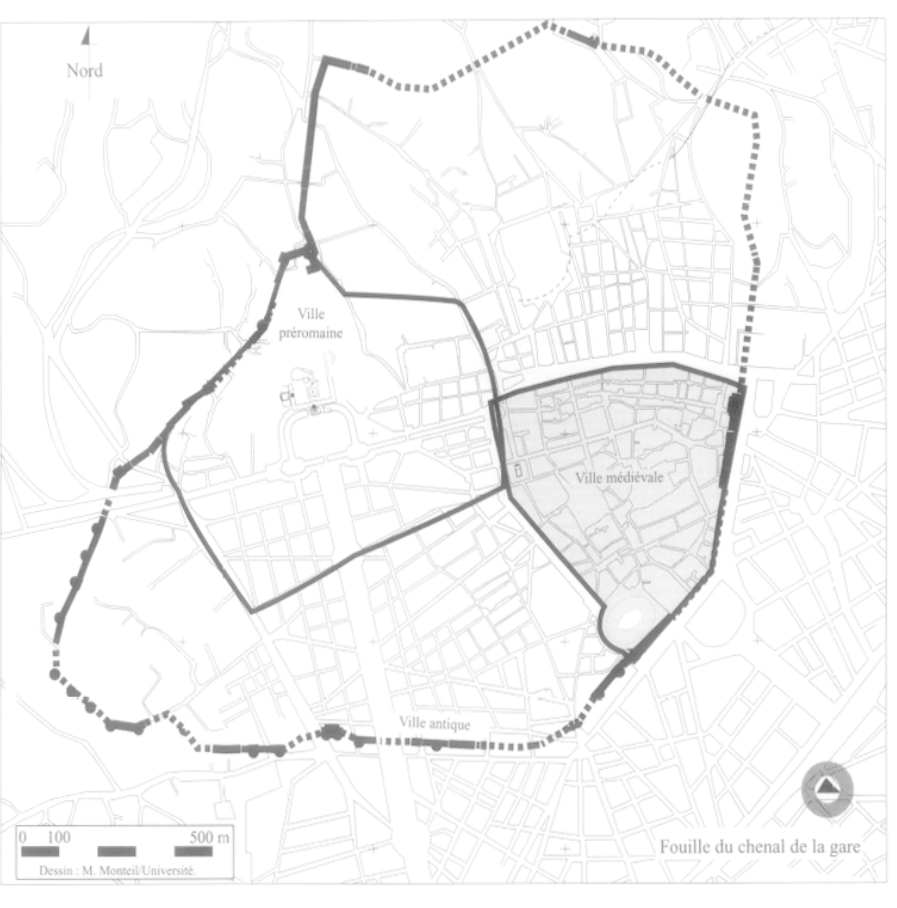

Fig. 2. Situation de la fouille par rapport aux tracés anciens de la ville de Nîmes (dessin M. Monteil, Université).

Latéralement, il est situé entre le cadereau d'Alès, à l'ouest, et le Vistre de la Fontaine, à l'est, à quelque $250 \mathrm{~m}$ de ce dernier (fig. 1). À l'angle de la rue Sainte-Félicité et du boulevard Natoire où nous l'avons observé, il suit une direction nord-ouest/sud-est, mesurée sur les $15 \mathrm{~m}$ de longueur de berge mis au jour. Cette orientation correspond à celle globalement suivie par les cadereaux du piémont nîmois et de la plaine du Vistre auxquels il est parallèle. En outre, il est en aval du piémont des Garrigues, dans la zone de contact formant un glacis en pente douce entre les collines calcaires et la plaine. Ce glacis est recouvert par des formations superficielles datées du Pléistocène, qui correspondent à un complexe de dépôts associant des fragments calcaires anguleux et cimentés (localement nommé sistre) à un matériel limoneux d'origine losssique. Le "sistre", présent sous la ville de Nîmes, constitue le substratum du secteur de la gare. Par endroits, un paléosol brun est conservé sur ce substrat. Il a été observé à deux reprises à proximité du chenal: au nord, à $70 \mathrm{~m}$ de distance (Manniez 2000, 8, 36) et au nord-est à $180 \mathrm{~m}$ (site de la ZAC de la gare III. Maufras 2000, 7, 8). Sa formation est antérieure au Néolithique final: en effet, aux portes de la ville, rue Servie, le paléosol est recoupé par des aménagements agricoles du Haut Empire (Monteil 1999, 58); sur le site de ZAC de la gare III, il est associé à du mobilier des $\mathrm{VI}^{\mathrm{e}}-\mathrm{III} \mathrm{I}^{\mathrm{e}} \mathrm{s}$. av. n.è. et dans la plaine du Vistre il est ponctuellement surmonté de couches du Néolithique récent (Chevillot 1999, 43).

3 "Cadereau" est le nom local donné à ces vallons pierreux qui charrient l'eau et les débris rocheux (Poupet et al. 1993, 30, note 6). 


\subsection{Le contexte historique}

La découverte du paléochenal est localisée à $1200 \mathrm{~m}$ au sud de la ville protohistorique et à $550 \mathrm{~m}$ des enceintes antique et médiévale de Nîmes, dans un secteur largement rural durant toutes ces périodes (fig. 2). Les établissements agricoles y sont encore clairsemés sur de vastes parcelles en 1829 quand est dressé le cadastre napoléonien (fig. 3). Le quartier s'est urbanisé après l'installation de la gare, dans la seconde moitié du XIX ${ }^{\mathrm{e}}$ s. et au cours du $\mathrm{XX}^{\mathrm{e}}$ siècle.

Les découvertes de vestiges anciens dans le secteur restent peu nombreuses et assez distantes du site de la gare. Elles permettent néanmoins de dresser le cadre général de l'occupation pendant l'Antiquité et le Moyen Âge.

À $550 \mathrm{~m}$ de la ville romaine, l'occupation n'est déjà plus périurbaine mais franchement rurale. Au nord du site, le boulevard Sergent Triaire et le Boulevard Talabot marquent grossièrement la limite entre un secteur proche des remparts où les découvertes sont relativement nombreuses et un secteur méridional où les trouvailles sont sporadiques (Fiches, Veyrac dir. 1996, 424-434) (fig. 4). Les vestiges bâtis les plus proches du site qui pourraient être rattachés à un habitat antique se trouvent à $150 \mathrm{~m}$ à l'ouest de celui-ci (fig. $4, \mathrm{n}^{\circ} 474$ ). À quelque $250 \mathrm{~m}$ de distance au nord-ouest, au nord et au nord-est, ce sont les découvertes funéraires qui dominent. Elles témoignent de la densité des nécropoles entre les voies de Saint-Gilles (rue Saint-Gilles) et d'Arles (rue Séguier). Cependant, la mise au jour de fosses de plantation (viticoles quand leur nature est identifiée) dans ces mêmes secteurs montre qu'ils étaient aussi cultivés (Monteil 1999, 42, 58). Plus au sud, les découvertes ne concernent plus qu'exceptionnellement des vestiges bâtis. Elles correspondent massivement aux vestiges en creux de la pratique agricole. Sur le site de la gare, des fossés qui limitent les parcelles antiques et une vigne ont été reconnus (Manniez 2000, 8-9) (fig. 4). Ils sont approximativement orientés à NL $38^{\circ}$ ouest et entre $\mathrm{NL} 23^{\circ}$ et NL $30^{\circ}$ ouest ${ }^{4}$, ce qui ne les rattache à aucun système parcellaire antique connu. Parmi les parcellaires nîmois, celui dont l'orientation est la plus proche est le "système vert" de J. Benoit, orienté à NL $33^{\circ}$ ouest (Benoît 1981, 75-86) que M. Christol et C. Goudineau proposent de dater de la fin de la République (Christol, Goudineau 1987, cités dans Monteil 1999, 32). Dans ce secteur, les seuls vestiges bâtis antiques consistent en un important collecteur, construit selon une direction nordsud et découvert à $55 \mathrm{~m}$ à l'est du paléochenal de la gare (Fiches, Veyrac dir. 1996, 430-431) (fig. 4, n 473). Comme ce dernier, il semble avoir contribué aux écoule-

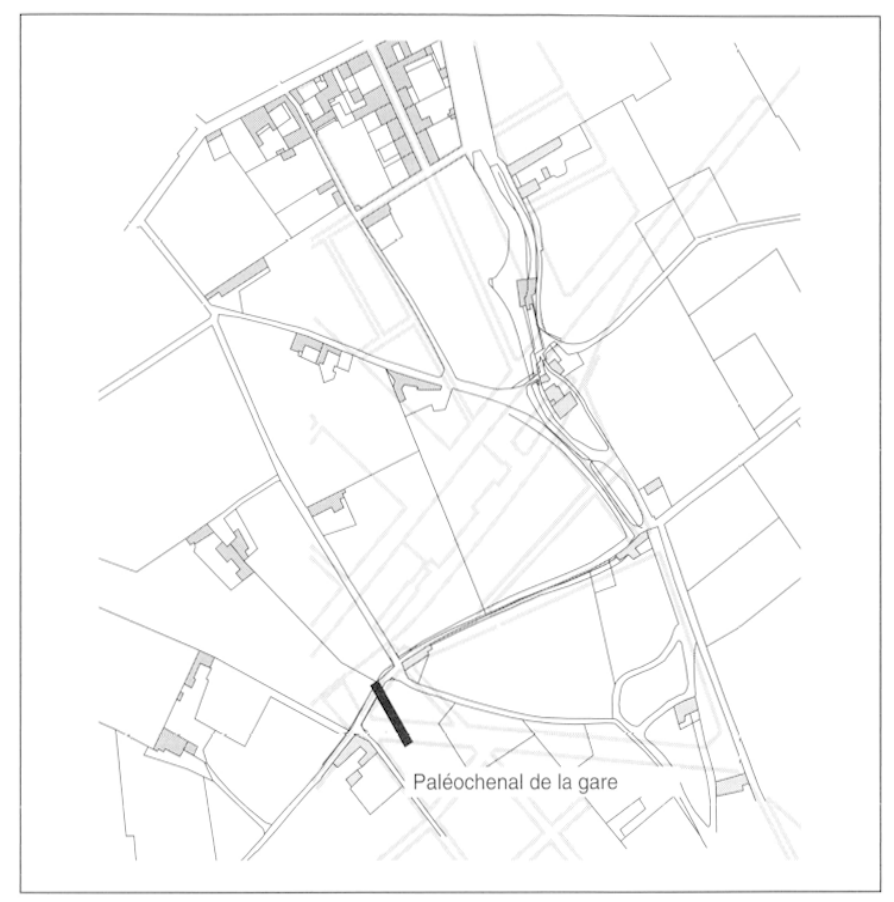

Fig. 3. Superposition des cadastres napoléonien (en trait noir), actuel (en trait grisé) et de l'emplacement du chenal de la gare (dessin Y. Manniez, Inrap).

ments d'eau de la ville vers le Vistre, mais sa date reste incertaine.

$\mathrm{Au}$ Moyen Âge l'occupation reste rurale. Si aucune découverte archéologique ne l'illustre, les textes du cartulaire de Nîmes le confirment. Ils mentionnent ante ipsa civitate ou bien, foras ipsa civitate ou encore ante porta, termes qui désignent les quartiers ruraux au sud de la ville (Mercier à paraître), des terres vouées à la culture des céréales, des pâturages, des vignes et dans une moindre mesure des oliviers (Germer-Durand 1874). Les terrains qui entourent le chenal de la gare sont probablement dans le territoire de la villa Vinisolo dont l'habitat principal est cité par les textes médiévaux au sud de son église, SaintePerpétue (Mercier à paraître), elle-même localisée à $375 \mathrm{~m}$ au nord-est de notre fouille (Fiches, Veyrac dir. 1996, 424) (fig. 4, n 457). Sainte-Perpétue est une basilique funéraire de l'Antiquité tardive mentionnée comme paroisse au $\mathrm{XI}^{\mathrm{e}} \mathrm{s}$. Le territoire de la villa compte au $\mathrm{X}^{\mathrm{e}} \mathrm{s}$. deux habitats groupés (villa Vinisolo et villare Gracmaco), peut-être trois au siècle suivant et sans doute plusieurs établissements agricoles à l'écart: un manse est mentionné en 936 à environ $1 \mathrm{~km}$ au sud-est de Sainte-Perpétue (Mercier à paraître). 
Fig. 4. Compilation d'extrait de la carte archéologique de Nîmes (Fiches, Veyrac 1996) et des découvertes récentes (dessin O. Maufras, Inrap).

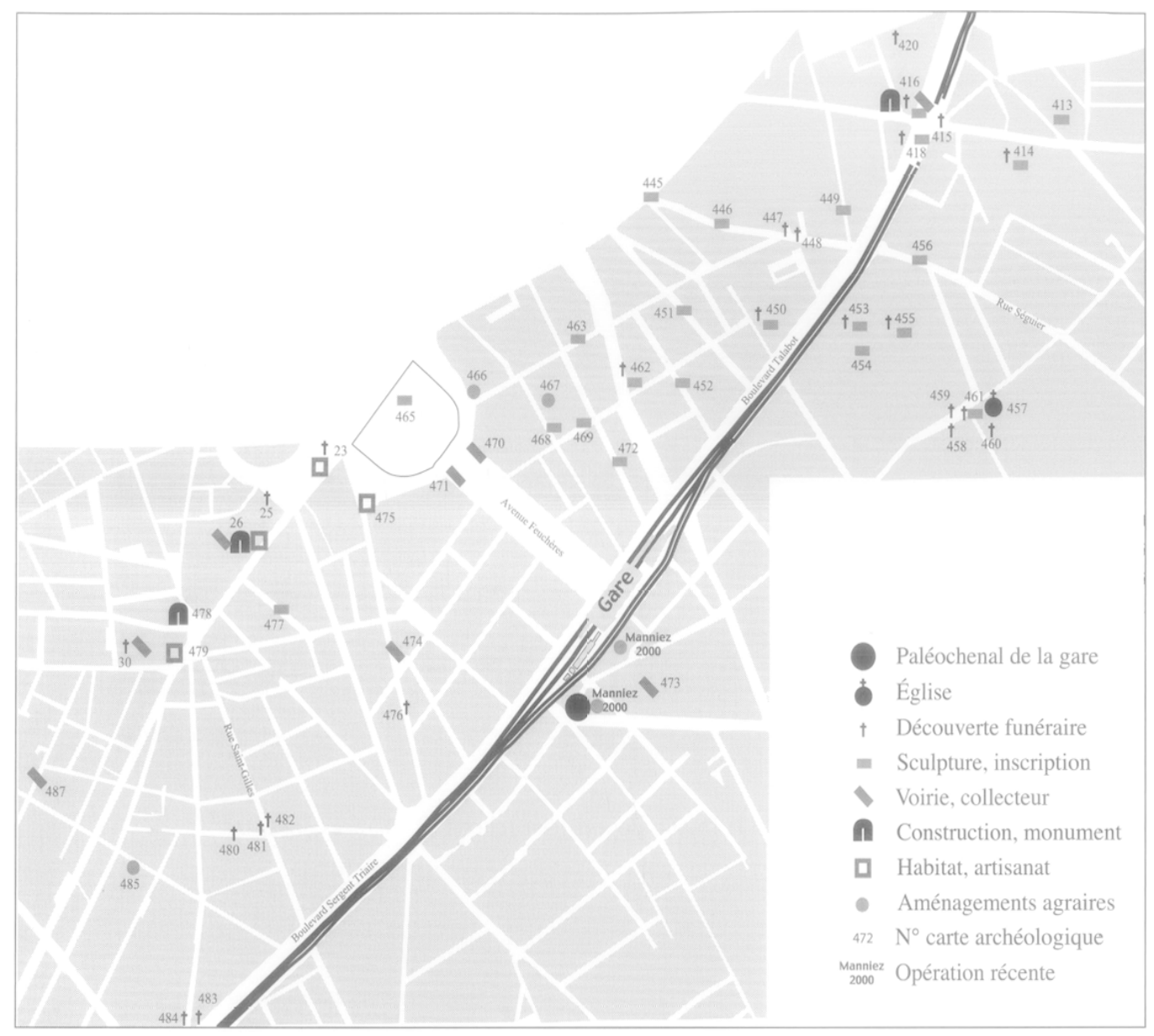

\section{La fouille du chenal}

L'opération archéologique, si elle a permis de percevoir la dynamique du chenal dans ses grandes lignes et d'appréhender l'évolution du milieu ainsi que l'activité humaine à l'entour, a été limitée par trois circonstances. La première vient de l'emprise de la fouille qui n'a pas touché le chenal dans toute sa largeur, la seconde de la rareté des mobiliers dans les couches les plus récentes, limitant l'établissement de la chronologie, la troisième de la pauvreté de certains échantillons paléoenvironnementaux.

L'ancien chenal de la gare a été reconnu au cours d'un diagnostic mené en 2000 (Manniez 2000) puis fouillé quelques semaines plus tard. L'opération archéologique a été limitée à la parcelle sise à l'angle des rues SainteFélicité, Quatrefages et du boulevard Natoire qui recoupe le cours d'eau sur environ $250 \mathrm{~m}^{2}$ : le chenal a donc fait l'objet d'une observation partielle (fig. 5, 6). En surface il a été décaissé sur toute sa superficie accessible, mais, du fait d'une excavation en paliers, il n'a été dégagé à la base que sur $2 \mathrm{~m}$ de largeur. La fouille a touché la rive orientale du cours d'eau, sur environ $15 \mathrm{~m}$ de long et $16 \mathrm{~m}$ de large. Ainsi la largeur totale reste inconnue et le fond atteint n'est peut-être que celui de la bordure et non du centre du chenal (fig. 6). La lecture des incisions et des comblements est donc incomplète. Deux lits successifs ont été observés sans que l'on sache s'il en existe d'autres et donc sans que l'on puisse restituer tous les ajustements de largeur et de profondeur, voire les déplacements que le cadereau a pu subir en fonction des variations de ses flux et de leur charge. Les comblements supérieurs ont été étudiés dans de bonnes conditions. En revanche, le colmatage le plus profond (004) n'a été observé jusqu'au substrat qu'au cours de son dégagement à la pelle mécanique, au travers de l'eau de la nappe phréatique, haute et abondante au moment de la fouille. Les matériaux qui constituent cette couche et son mobilier ont fait l'objet de ramassages au fur et à mesure que la pelle mécanique les déposait sur la berge. La berge elle-même n'était conservée que sur une faible distance, le long du chenal, mais pas sur toute sa hauteur. Beaucoup plus haute que le lit du cours d'eau, elle a souffert d'une troncature due à la mise en culture et aux aménagements de l'époque moderne. Par ailleurs, il n'a pas été possible de réaliser de coupe strictement perpendiculaire au cadereau, si ce n'est en deux parties (fig. 7, 8).

Le mobilier récupéré dans les différentes couches est d'un intérêt inégal. Les comblements profonds ont fourni quelques niveaux riches d'un mobilier homogène qui permet d'établir dans ses grandes lignes la chronologie abso- 


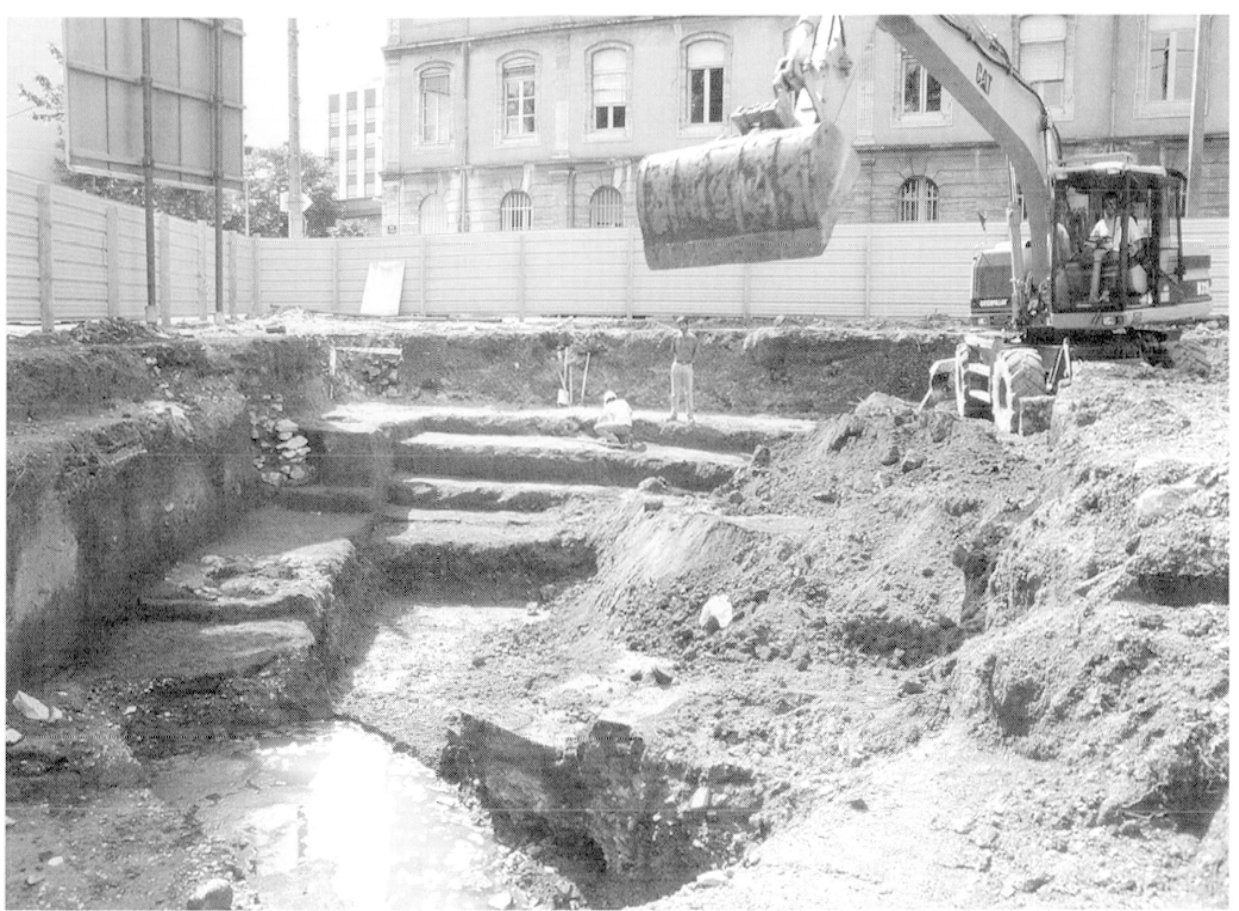

Fig. 5. Vue d'ensemble de la fouille depuis l'est (cliché O. Maufras, Inrap).

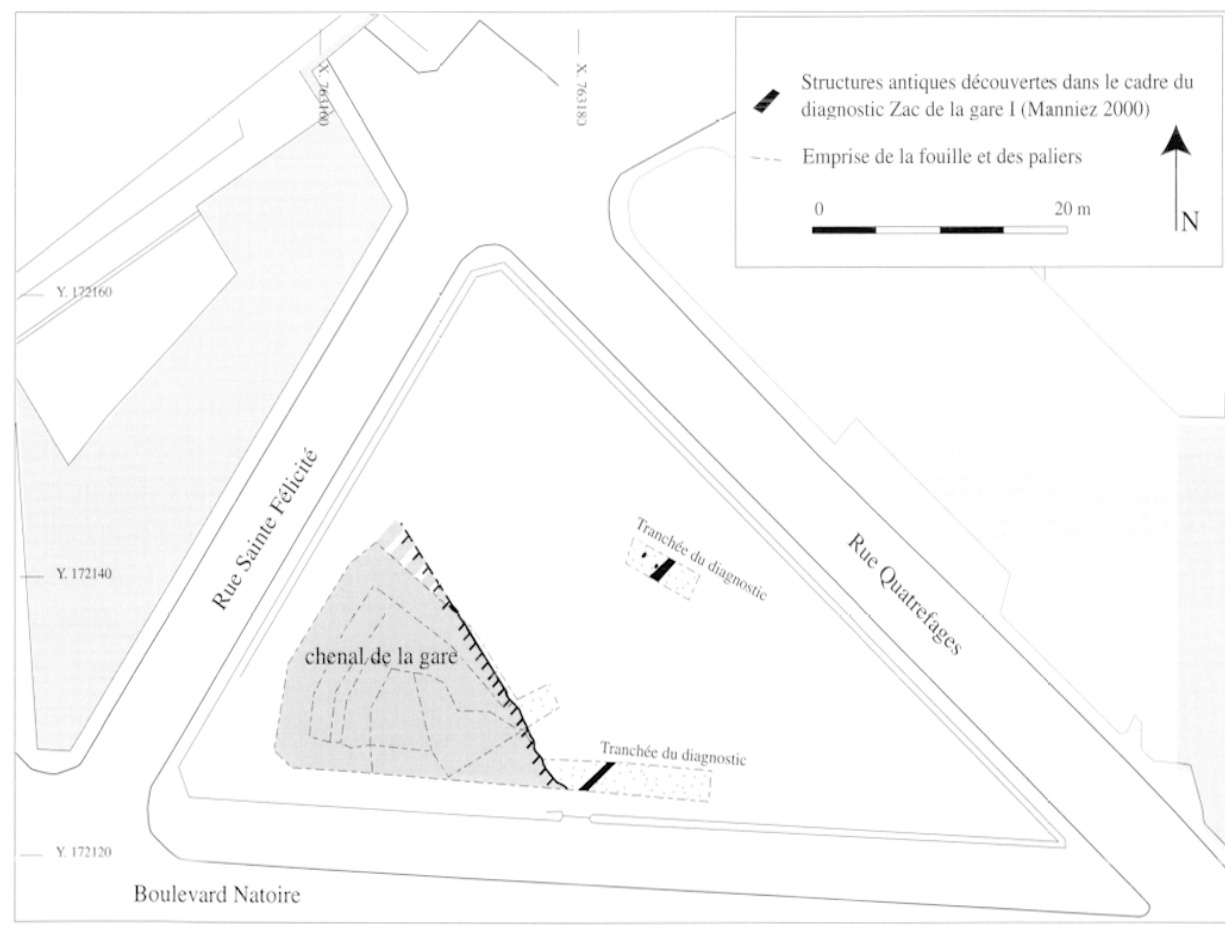

Fig. 6. Plan des découvertes archéologiques: paléochenal et proches fossés antiques (dessin C. Bioul; V. Lelièvre, Inrap). lue des dépôts successifs. Les niveaux supérieurs par contre, et en particulier les colluvions, n'ont livré qu'un matériel parcimonieux qui semble provenir d'un site antique voisin dont les vestiges, ont été continuellement érodés. Ainsi les comblements les plus récents du chenal contiennent-ils massivement du mobilier des ${ }^{\text {er }}$ et II $^{e} s$. de n.è., bien qu'ils se soient mis en place entre cette date et l'époque moderne. À partir du III ${ }^{\mathrm{e}} \mathrm{s}$. nous ne disposons donc plus que de la chronologie relative des événements stratigraphiques.

Enfin, toutes les études environnementales initialement prévues n'ont pu être mises en œuvre. Ainsi, faute de graines et de charbons conservés en nombre suffisant dans les différentes couches du site, les analyses carpologiques, anthracologiques et entomologiques ont été abandonnées. L'étude palynologique a été limitée à l'exploitation de sept échantillons prélevés dans les couches supérieures bien qu'ils se soient avérés pauvres ${ }^{5}$. En revanche, les prélèvements malacologiques, micromorphologiques et granulométriques illustrent l'ensemble de la stratigraphie, à l'exception toutefois du niveau profond fouillé mécaniquement et pour lequel les risques d'interférence étaient trop lourds. Ces études ont été menées en complément des observations archéologiques et géomorphologiques de terrain qu'elles ont largement précisées.

En dépit de ces limites, les différentes approches se complètent pour dresser l'évolution de

5 Les couches les plus basses subissant le battement de la nappe phréatique et la probable migration verticale des particules fines, elles n'ont pas fait l'objet de prélèvements. 
l'environnement dans ses grandes lignes. La chronologie relative des événements est bien cernée. Les analyses sédimentaires ont permis une bonne identification de la nature des comblements et, couplées à l'étude de la malacofaune, elles ont permis la restitution de la paléohydrologie. Le milieu environnant et l'activité humaine ont été documentés par la micromorphologie des couches et tantôt par les coquillages qu'elles contenaient, tantôt par les pollens.

\section{Indices d'évolution du milieu dans le secteur de la gare du $I^{\text {er }} S$. av. n.è. à l'époque moderne}

\subsection{Un cadereau dynamique jusqu'à la fin du I'r s. de n.è.}

Jusqu'à la fin du I ${ }^{\text {er }} \mathrm{s}$. de n.è., le cadereau de la gare a connu un régime hydrologique dynamique et irrégulier. Des variations sont en effet perceptibles, qui traduisent des écoulements importants liés à des périodes d'activité fortement torrentielle où alternent incisions et alluvionnements. Les deux premières périodes sont documentées par l'observation visuelle des couches, la troisième bénéficie de l'étude granulométrique qui précise la nature et l'intensité des flux.

\section{UN HYDRODYNAMISME ACTIF ET VARIABLE}

L'activité hydrologique la plus ancienne qui ait été identifiée est marquée par l'incision d'un lit (053) et les dépôts alluviaux qui l'ont progressivement colmaté (061 puis $060=070$, fig. 7).

Le creusement 053 du premier chenal perce le sistre, 073, semble-t-il antérieurement à la formation du paléosol brun observé lors de l'opération de diagnostic (Manniez 2000). Cependant, cette chronologie n'a pas été confirmée, le contact physique entre le chenal et le paléosol n'étant conservée nulle part sur l'emprise de la fouille. Le cadereau présente un bord oriental rectiligne incliné de $20^{\circ}$ environ, conservé sur $1,65 \mathrm{~m}$ de haut. Son fond est presque plat et se trouve lui-même conservé sur $2,25 \mathrm{~m}$ de large. Cette incision présente un profil étonnant: on s'attend, dans un encaissant aussi résistant que le sistre, à un lit aux parois très évasées. Cependant on hésite à conclure à un aménagement volontaire (une régularisation du bord du cours) d'autant que des cadereaux supposés naturels à fond plat ont été reconnus à Nîmes ${ }^{6}$.

Les premiers comblements du chenal, 061 puis 060 $=070$ (fig. 7,8$)$, procèdent d'apports multiples (non indi- vidualisés à la fouille) de matériaux naturels classés: graviers principalement et sables correspondant à différentes crues. La charge varie d'un apport à l'autre: certaines couches graveleuses contiennent des cailloux, d'autres non, quelques poches sont plus riches en sables, voire essentiellement constituées de sables et de limons. Les charges véhiculées dans le chenal sont grossières. Elles dénotent l'importance des flux hydriques sans que leur rythme puisse être mesuré ?

La seconde phase de l'activité alluviale du cadereau est marquée par un hydrodynamisme plus fort, illustré par une nouvelle incision puis des dépôts plus grossiers.

Au moment de ces nouvelles crues, le premier lit, partiellement ou entièrement colmaté, fait l'objet d'un nouveau creusement (064), décalé par rapport au premier (sur l'emprise de la fouille) de $2,95 \mathrm{~m}$ vers l'ouest. Le second lit du chenal est plus profond, $1,20 \mathrm{~m}$ plus bas que le précédent. Son profil est similaire à celui du lit antérieur, avec un fond presque plat et une paroi inclinée (fig. 7). Cette reprise de l'incision du lit marque un accroissement de la puissance du flux hydrique que l'on constate également par la charge du comblement inférieur du nouveau chenal. En effet, les couches 004 et 069 sont constituées de galets roulés de 5 à $12 \mathrm{~cm}$ de long qui atteignent parfois $20 \mathrm{ou}$ $25 \mathrm{~cm}$. À $6 \mathrm{~m}$ du bord oriental du chenal, le dépôt $004 \mathrm{se}$ développe sur $1,70 \mathrm{~m}$ de hauteur. La grossièreté des alluvions, sans matrice fine, traduit des dynamiques alluviales de type torrentiel, capables de transporter des charges solides loin des zones d'approvisionnement.

Au-dessus, et marquant une troisième phase dans l'activité du chenal, les dépôts alluviaux se poursuivent $(048$, 045, 040, 041 et 046, 042 et 047, 050, 043, 067, 057, 049, 044). Bien qu'ils présentent de nouveau une texture moins grossière, ils témoignent encore de crues à fort régime. Les apports 048, 045, 040, 041, 046 et 043 présentent les caractéristiques des dépôts forcés de type torrentiel: texture gravillonneuse et sableuse avec un mode centré autour de $1 \mathrm{~mm}$. Leur courbe granulométrique cumulative est de type hyperbolique (forme convexe bien redressée) ( $c f$. infra annexe 1). Il est intéressant de noter que ce fonctionnement torrentiel caractérise généralement les cônes de déjection ou l'amont de petites zones de delta. Nous sommes donc en présence de séquences alluviales de crue à fort régime capables de déposer des charges de fond grossières, les particules fines étant quant à elles véhiculées plus loin vers l'aval.

6 G. Fabre et $P$. Poupet les évoquent, mais aucune coupe n'est publiée dans les ouvrages auxquels nous avons eu accès.

7 Ces niveaux ont été fouillés dans un étroit sondage et rapidement observés avant la remontée de la nappe phréatique. Celle-ci étant intervenue en quelques dizaines de minutes, nous n'avons pas eu le temps de faire de prélèvements. 


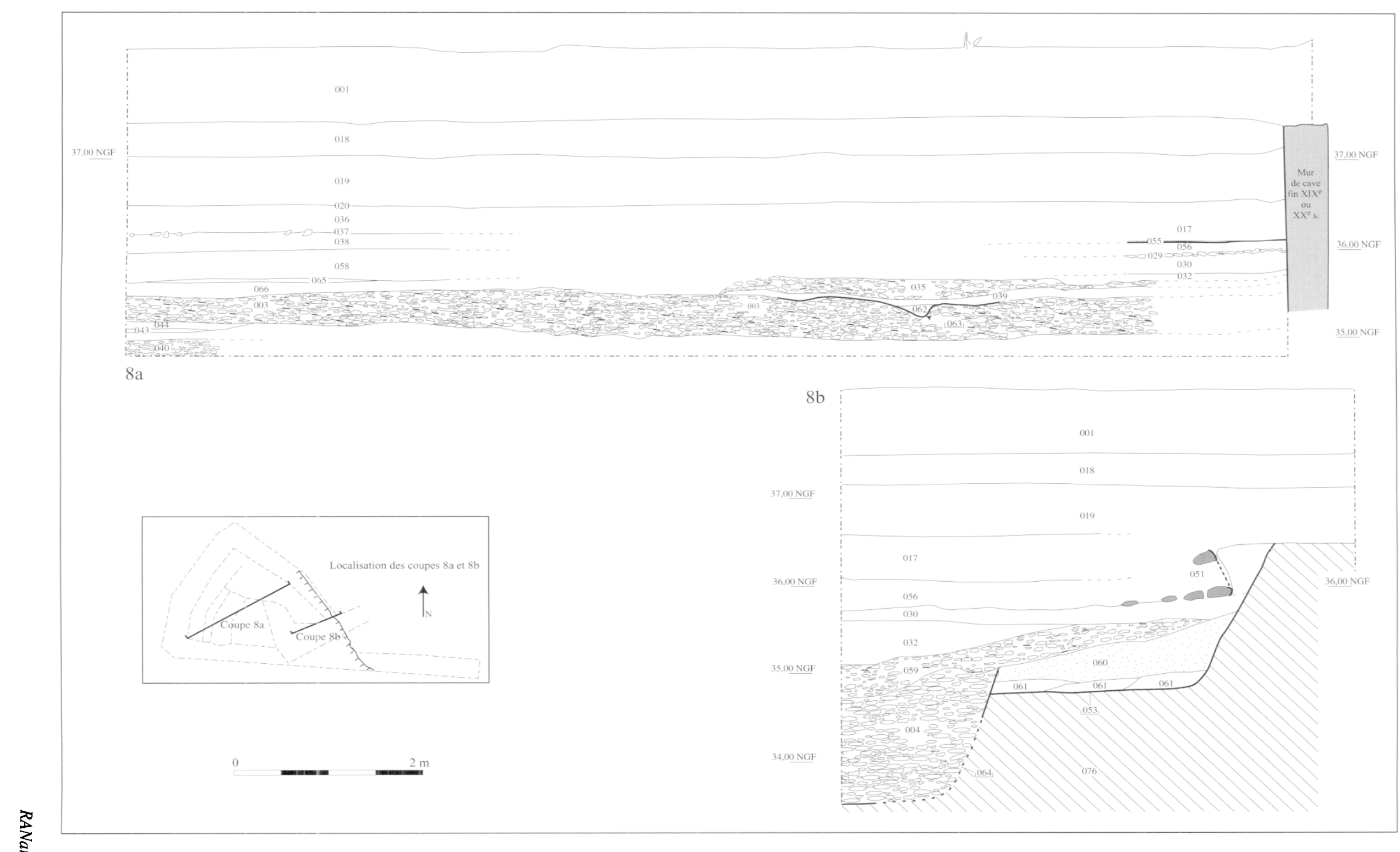

Fig. 7. Coupes perpendiculaires à l'axe du chenal. $8 \mathrm{a}$ : coupe nord-est/sud-ouest de la partie centrale du chenal. $8 \mathrm{~b}$ : coupe nord-est/sud-ouest du bord oriental du chenal (dessin C. Bioul; O. Maufras, Inrap). 
Au sommet de cette séquence, certaines couches accusent un ralentissement du flux: l'apport 050 montre une courbe cumulative des sables particulièrement significative d'une dynamique alluviale à régime modéré (courbe à profil en S. Cf. infra annexe 2). Sa texture est sablo-limoneuse, les sables grossiers y sont prédominants et l'histogramme présente un mode bien marqué à 500 microns. L'apport 047 qui s'intercale entre les niveaux de crue 046 et 043, et le dépôt 049 sont significatifs de dépôts de fin de crue où la baisse du courant permet aux particules fines de se déposer, notamment les mollusques (observés dans la couche 057 ) dont les coquilles se trouvent toutefois très corrodées par le transport (cf. infra annexe 3). Ces dépôts se caractérisent par une texture limono-sableuse dans laquelle la fraction sableuse est composée pour plus de $50 \%$ par des sables fins. Ce sont des niveaux très minces et discontinus car ils sont en grande partie érodés lors de la crue suivante.

\section{LES INDICES DE DATATION}

Ces trois phases actives de l'histoire du cadereau sont antérieures à la fin du Ier $\mathbf{s}$. de n.è. Les deux premières sont datées chacune par un lot homogène de mobilier véhiculé avec l'une des charges qui les composent: 070 et 004 . La troisième est calée chronologiquement par la seconde qui offre un terminus post quem et le terminus ante quem fourni par l'aménagement du cadereau qui la surmonte ( $c f$. infra $\$ 3.2$ ).

Le dépôt 070 qui comble la première incision a fourni six tessons provenant d'un minimum de trois objets: une amphore italique, une cruche à pâte claire récente de type $3 \mathrm{a}$ ou $3 \mathrm{~b}$ et une urne modelée de type U5. Cet ensemble peut être rattaché à la période républicaine même si nous raisonnons sur un échantillonnage restreint. L'amphore italique, diffusée à partir de la deuxième moitié du $\mathrm{II}^{e} \mathrm{~s}$. av. n.è. (Py 1993, 53) côtoie une forme de cruche en céramique à pâte claire récente produite à partir du dernier quart du II' ${ }^{\mathrm{e}}$ s. (Py 1993, 226) et un bord d'urne en céramique modelée de tradition protohistorique. La présence de fragments de tuiles montre que cette couche s'inscrit dans le courant du I ${ }^{\mathrm{er}} \mathrm{s}$. av. n.è. puisque ces éléments d'architecture apparaissent dans l'habitat domestique local à partir du deuxième tiers de ce siècle (Py 1990, 213)

L'unité stratigraphique 004 a fourni 5 fragments de céramique provenant d'une amphore de Tarraconaise, d'une amphore italique et d'une ou deux cruche(s) à pâte claire récente. Seul un terminus post quem peut être assuré pour cette couche qui semble s'être mise en place une cinquantaine d'années après la précédente. En effet, elle contient une panse d'amphore de Tarraconaise, production qui est diffusée à partir du milieu du I ${ }^{\text {er }} s$. av. n.è. en Narbonnaise (Fiches 1994, 337-341). Le reste du mobi- lier céramique ne contredit pas une datation qui pourrait être centrée dans la deuxième moitié de ce siècle, voire au cours du suivant.

La première incision du cadereau reste donc indatable. En revanche, elle reçoit des dépôts dont l'un remonte au $\mathrm{I}^{\mathrm{er}} \mathrm{s}$. av. n.è. Il est recoupé par le creusement du chenal de la deuxième phase dont l'un des comblements identifiés s'est formé entre le milieu du I ${ }^{\text {er }} s$. av. n.è. et le courant du $\mathrm{I}^{\mathrm{er}} \mathrm{s}$. de n.è. La troisième phase s'est déroulée avant l'aménagement d'un chemin dans le cadereau, construit entre la fin du Ir $s$. et le milieu du II . Quel que soit le moment de sa mise en place, le chenal s'est donc trouvé à moitié colmaté entre la fin du $\mathrm{II}^{\mathrm{e}} \mathrm{s}$. av. n.è. au plus tôt et le milieu du $\mathrm{II}^{\mathrm{e}} \mathrm{s}$. de n.è. au plus tard. Cette rapidité est le signe d'un changement brutal du milieu sous l'effet, soit d'une oscillation climatique, soit de la pression des sociétés galloromaine sur les secteurs en amont.

\section{LE PAYSAGE ENVIRONNANT}

L'environnement du cadereau de la gare au cours du $\mathrm{I}^{\mathrm{er}} \mathrm{s}$. av. et du $\mathrm{I}^{\mathrm{er}} \mathrm{s}$. de n.è. est documenté par les coquilles d'un échantillon malacologique et de rares vestiges archéologiques découverts dans les environs.

Le dépôt alluvial 057 a livré, outre des coquilles aquatiques, un ensemble d'une dizaine d'espèces terrestres qui affectionnent les milieux ouverts ( $c f$. infra annexe 6). Cela suggère un environnement largement déboisé et sans doute déjà voué à la culture ou au pâturage.

$\mathrm{Au}$ contact du cadereau, sur la berge orientale et sur un peu plus de $100 \mathrm{~m}$, deux étapes d'occupation agricole du secteur ont été mises en évidence. Une vigne dont les rangs de fosses oblongues sont orientés entre NL $23^{\circ}$ et NL $30^{\circ}$ ouest a livré un mobilier parcimonieux de l'époque républicaine, peut-être contemporain des dépôts de crues torrentielles du premier chenal (Manniez $2000,8,24)$. Elle est recoupée par un parcellaire dont l'orientation est sensiblement plus inclinée à l'ouest: le fossé qui traverse la vigne est orienté à NL $38^{\circ}$ ouest. Son comblement inférieur contient du matériel céramique du Haut Empire (Manniez 2000, 9, 24). Si les traces de l'occupation agraire du secteur livrent du mobilier des $\mathrm{I}^{\mathrm{er}} \mathrm{s}$. av. et du $\mathrm{I}^{\mathrm{er}} \mathrm{s}$. de n.è., l'organisation des terrains en culture n'en paraît pas moins plus ancienne. C'est ce qu'il semble à l'observation du paléosol sousjacent aux structures antiques. À quelque $225 \mathrm{~m}$ au nordouest du chenal de la gare, il contient, enfouis à plusieurs niveaux de la couche, du mobilier céramique produit entre le $\mathrm{VI}^{\mathrm{e}}$ et le $\mathrm{III}^{\mathrm{e}} \mathrm{s}$. av. n.è.: fragments d'amphore massaliète, de céramique non tournée et de dolium qui suggèrent un amendement du sol de ce secteur dès la protohistoire (Maufras 2000, 8). 


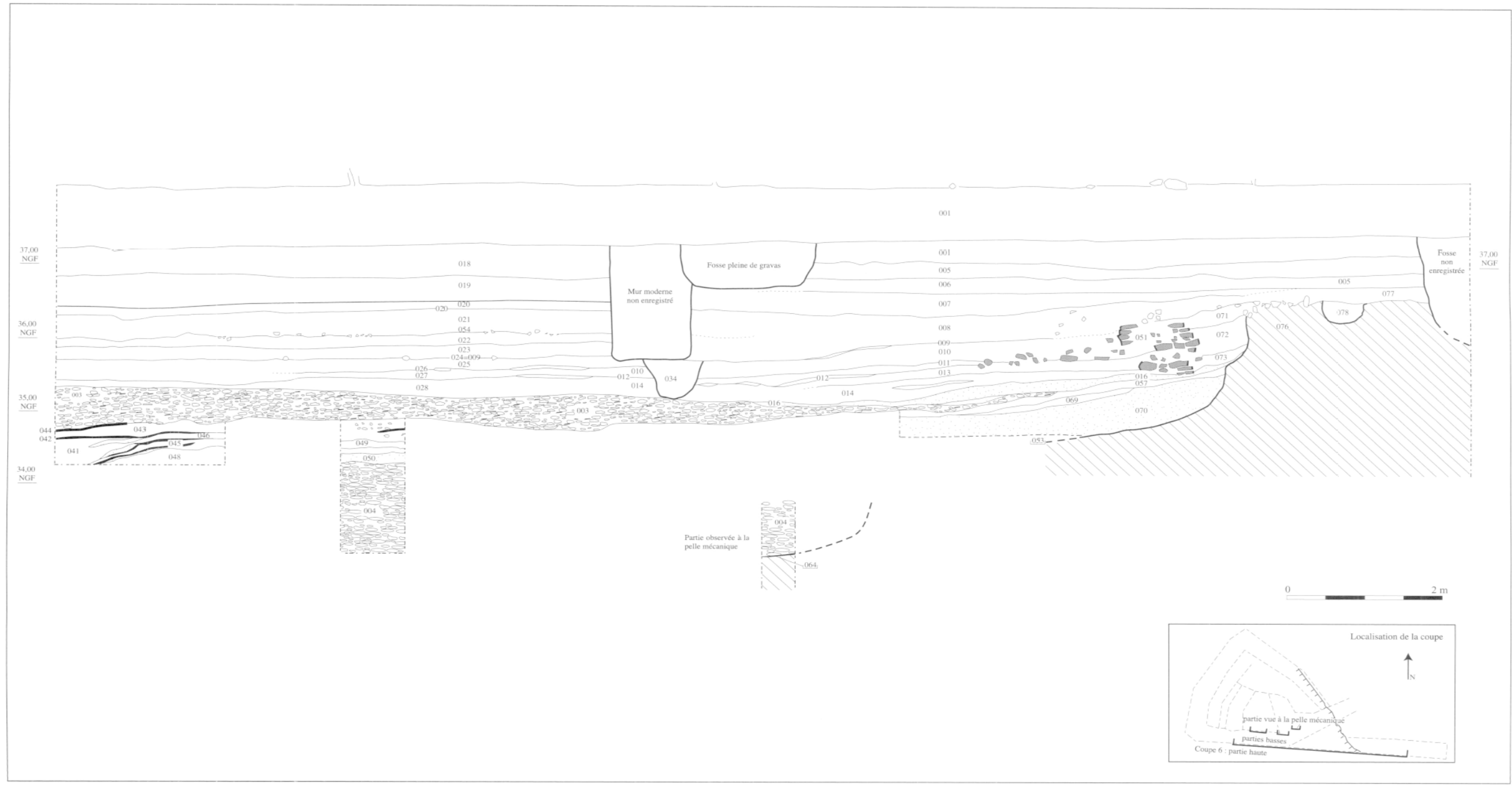

Fig. 8. Coupe est-ouest, en biais dans le chenal (dessin C. Bioul; P. Chevillot, O. Maufras, Inrap). 
Fig. 9. Plan des parties observées des trois principaux nivaux de circulation du chemin antique (dessin C. Bioul, Inrap; O. Maufras, Inrap).

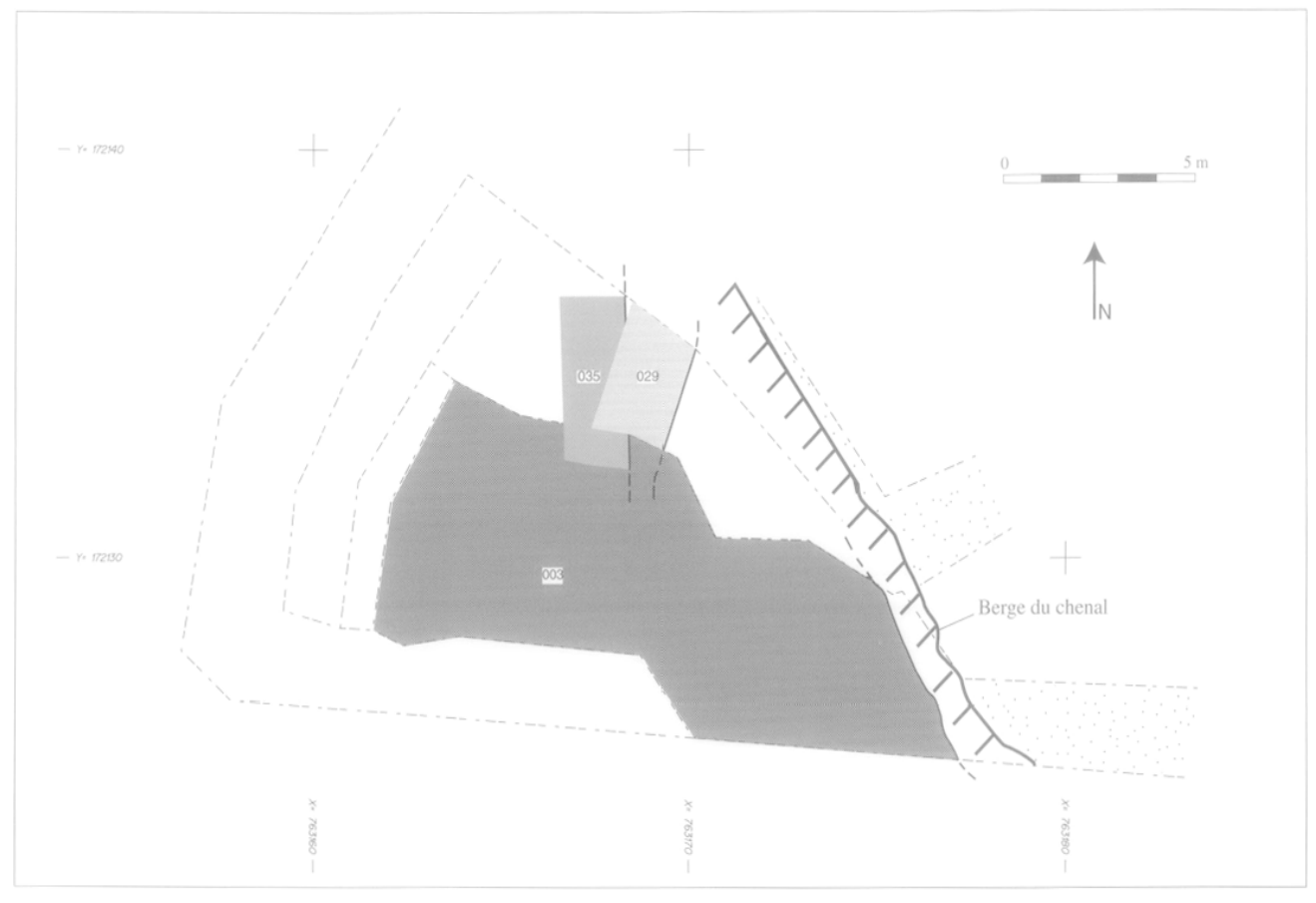

\subsection{L'aménagement du cadereau en voie terrestre}

Dans l'histoire du chenal de la gare, la période suivante n'est marquée par aucun changement hydrologique notable: on reste dans une phase de crues dont la puissance diminue progressivement. C'est l'intervention de l'homme qui marque une étape importante. Le lit du cadereau est en effet aménagé en chemin.

\section{DES FLUX HUMAINS}

ET DES FLUX LIQUIDES

L'aménagement du cadereau en voie de circulation est simple. Le fond du chenal est exhaussé d'une cinquantaine de centimètres par l'apport d'un remblai de matériaux grossicrs et donc drainants. Le remblai $(003=015$ $=033=059$ ) est constitué de plusieurs apports de pierres de toutes tailles, de nombreux éléments de construction, de fragments de vaisselle de table, de céramique de stockage et d'ossements animaux (fig. 7, 8). Il forme une chaussée quasiment plane, stable, qui a été dégagée sur $6,30 \mathrm{~m}$ de largeur (fig. 9). Une ornière a été observée (063, fig. 10) qui suit l'axe général du chenal, et plusieurs recharges ponctuelles ont été identifiées, faites également de matériaux hétérogènes et grossiers $(062,035,016$ et 029 , fig. 11). Après une première utilisation du chemin, son aménagement a été complété par la construction d'un mur de berge installé dans le chenal, le long de son bord oriental $(051=075)$. Celui-ci est une construction dou- blement parementée de $0,60 \mathrm{~m}$ de large environ, conservée sur huit assises de moellons de calcaire équarris et liés à la terre (fig. 12,13). Le mur a été bâti à quelques centimètres de la paroi du chenal, retaillée pour l'occasion. L'espace entre parement et paroi a ensuite été colmaté par les apports 073, 072 et 071 (fig. 8).

Entre les niveaux de recharges de voie, on note la présence de dépôts à granulométrie plus fine, d'origine naturelle $(039,032,031,030,014=028=066,013,012$ $=065$ et 011 ). Il s'agit de nouveau d'apports alluviaux. L'activité du chenal est notamment attestée par le fort pourcentage des coquilles d'espèces aquatiques retrouvées dans la couche $031(66,25 \%)$. Certaines de ces espèces sont tolérantes et supportent des périodes hors d'eau, mais Ancylus fluviatilis, Bathyomphalus contortus et Acroloxus lacustris nécessitent, les deux premiers, un milieu permanent d'eaux courantes, et le troisième une certaine profondeur d'eau stagnante ${ }^{8}$. Cet assemblage malacologique plaide pour l'existence d'un flux permanent ou bien, immédiatement en amont, d'une source ou d'une mare toujours en eau, qui expliquerait l'importance d'Ancylus fluviatilis et de Bathyomphalus contortus. La présence de Bythiospem acicula dans la couche 031, espèce souvent présente dans les sources, appuierait la seconde hypothèse tandis que le développement de Bathyomphalus contortus dans la couche 030 marquerait une eau plus stagnante, ralentie et riche en végétation. 


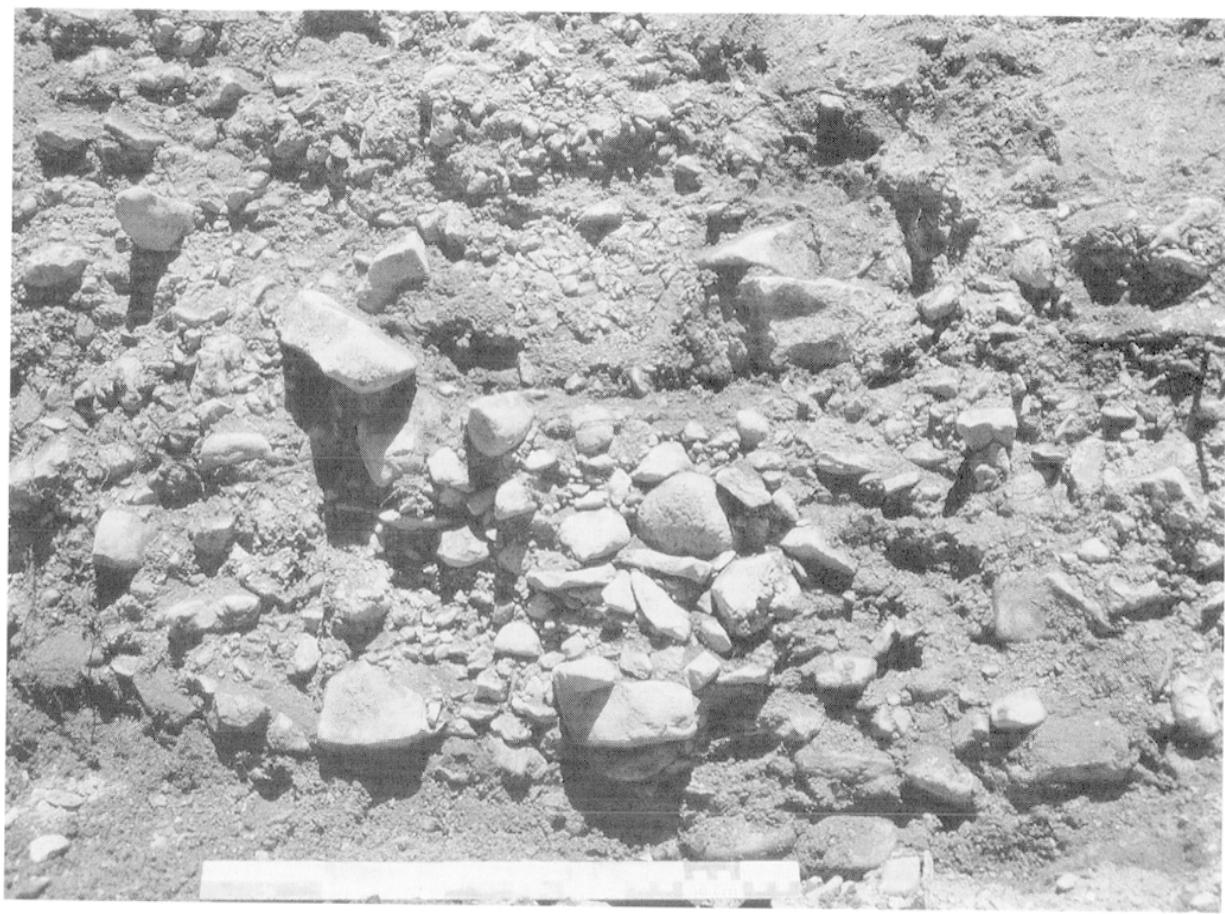

Fig. 10. Détail du niveau de voie 003 en coupe et de l'ornière 063 (cliché O. Maufras, Inrap).

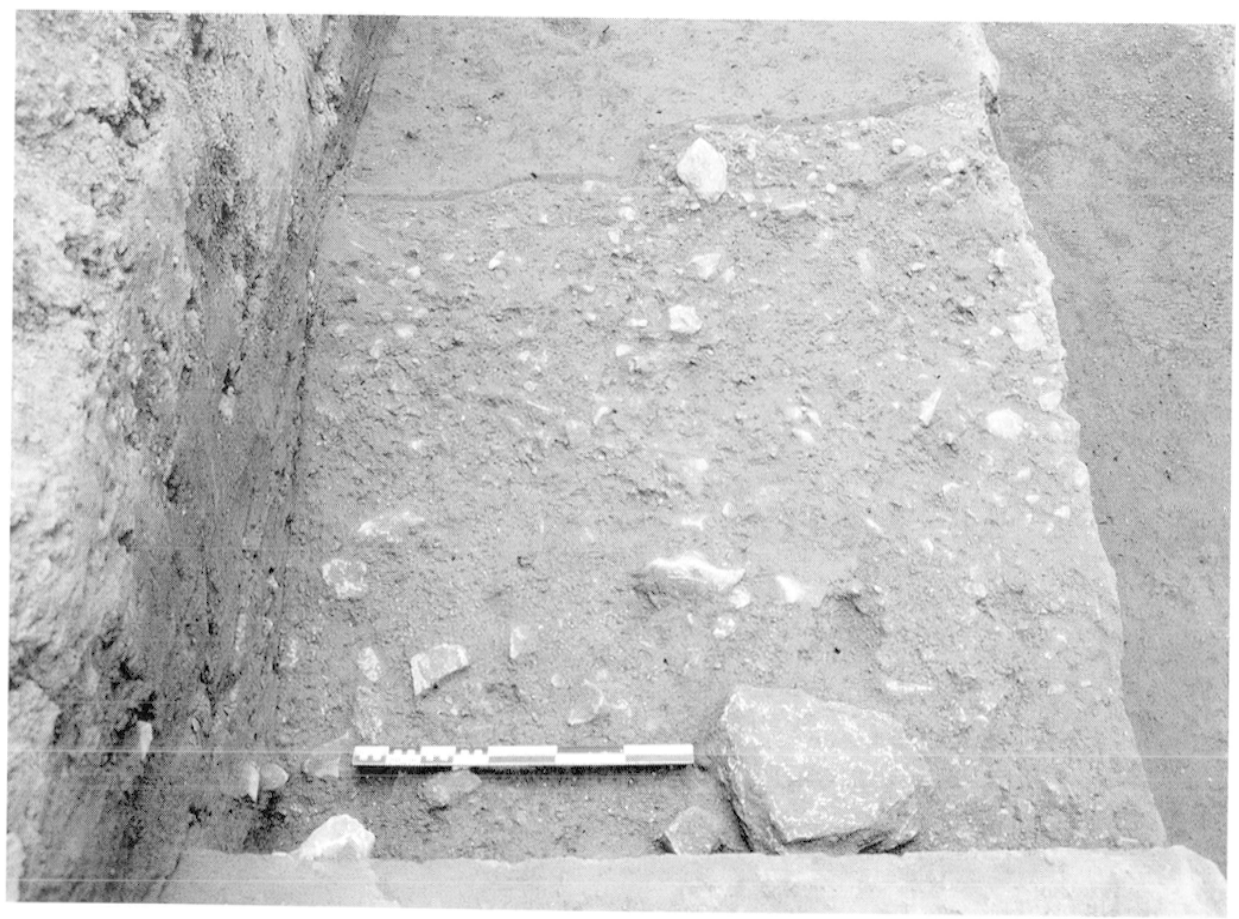

Fig. II. La recharge 029 sur le chemin (cliché O. Maufras, Inrap).
La bonne conservation des coquilles, en particulier de celles du fragile Ancylus fluviatilis, qui sont ni brisées ni corrodées, suppose que les flux qui transitent dans le cadereau transportent une faible charge, peu érosive, sur une faible distance. La présence dans les échantillons malacologiques des dépôts 031 et plus particulièrement 030 , d'espèces affectionnant la végétation abondante des eaux du chenal ou de ses marges plaide également pour des eaux à faible charge solide qui permettent le développement des plantes. L'analyse granulométrique confirme la diminution progressive du régime. À l'instar des apports 050, 047 et 049 de la fin de la phase précédente, les niveaux 031 et 030 traduisent des dynamiques alluviales de faible compétence. Il est possible également que les épisodes de crue aient été moins fréquents. Ainsi le chenal a-t-il assuré une double circulation: celle des flux hydriques vers la plaine et celle des hommes entre deux crues.

\section{LES INDICES DE DATATION}

Le remblai d'exhaussement du fond du chenal qui forme la chaussée de la voie $(003=015$ =033) a fourni 523 tessons de céramique provenant d'un minimum de 81 objets ${ }^{9}$, et quatre monnaies ${ }^{10}$. Quelques rares éléments remontent à la protohistoire ou à la période républicaine: amphore italique, amphore massaliète et céramique modelée. C'est le cas également de deux des monnaies, un as de la péninsule ibérique du ${ }^{\text {er }} \mathrm{s}$. av. n.è. et un as de Rome frappé sous Claude, mais il est fréquent de retrouver à Nîmes les monnaies d'argent plusieurs décennies après leur mise en circulation. Pour le reste, le lot est homogène et plus récent, il est datable des années 90-150. 
Fig. 12. Vue générale de la coupe réalisée en biais sur le paléochenal, notamment sur le mur de berge 051 (cliché O. Maufras, Inrap).

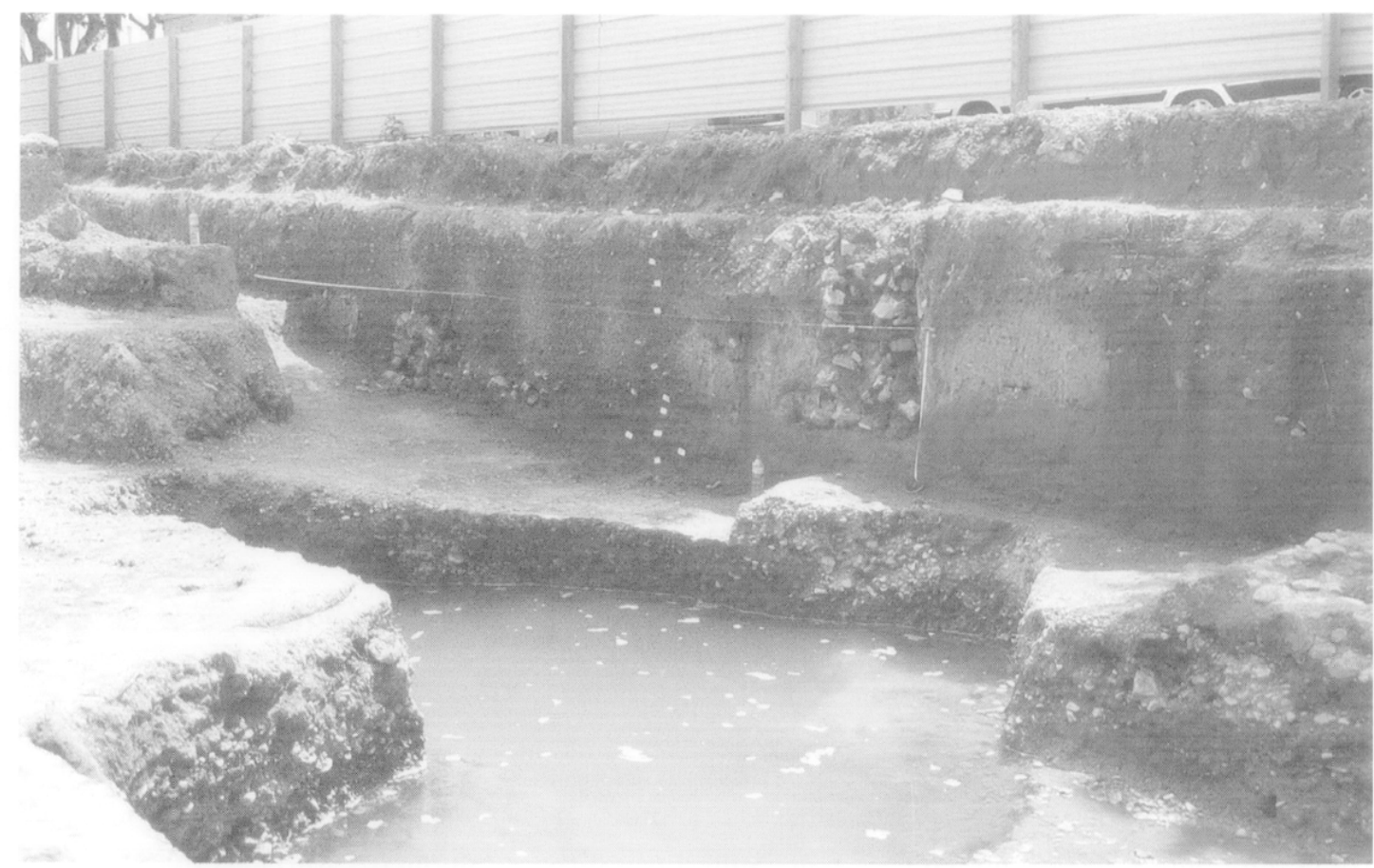

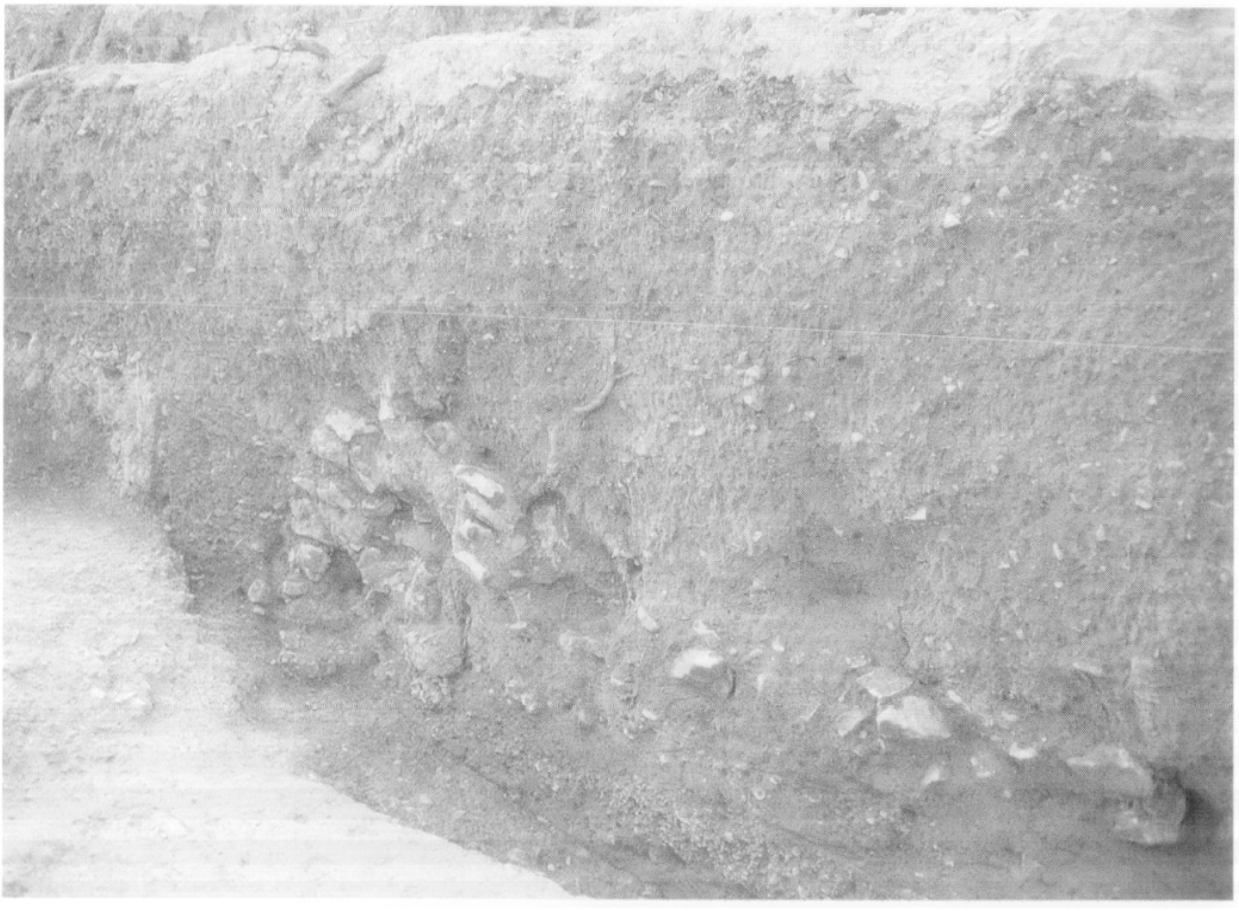

Fig. 13. Détail du mur de berge 05I sur la partie orientale de la coupe réalisée en biais sur le paléochenal (cliché O. Maufras, Inrap).
En effet, les deux deniers, monnaies de moindre valeur plus significatives pour un essai de datation, ont été frappés sous Domitien en 86 et en $88 / 89$ : ils fournissent un terminus post quem à la fin du $\mathrm{I}^{\mathrm{er}} \mathrm{s}$. Ce terminus peut être rajeuni d'une dizaine d'années au regard du mobilier céramique associé: la présence de céramique africaine de cuisine, illustrée par un bord de marmite de type Hayes 197 et deux formes de récipients en sigillée sud-gauloise, présents en plusieurs exemplaires (coupelle/assiette Drag. 35/36 et coupe Drag. 37), situe la constitution de la couche à partir de la période flavienne. Plus précisément, un terminus post quem vers 100 peut être avancé si l'on tient compte de l'existence de formes produites à partir de cette date: un bord de coupe de type Hayes 9a en claire A (Raynaud 1993a, 172) ou des urnes de type A7 et $\mathrm{A} 8$ en commune à pâte

9 Dans ce comptage, le mobilier de la couche $015 \mathrm{n}$ 'a pas été pris en compte. Il avait été isolé à la fouille du fait du risque d’interférence avec le mobilier d'une fosse moderne identifiée tardivement.

10 En revanche, bien que pouvant également être intrusive (cf. supra note 9), la monnaie de la couche 015 a été prise en compte. Toutes les monnaies ont été identifiées par M. Amandry (Cabinet des médailles, Paris). 
sableuse (Raynaud 1993c, 549-550). Nous n'envisagerons pas une datation au-delà du milieu du $\mathrm{II}^{\mathrm{e}} \mathrm{s}$. en raison de l'absence de la céramique claire $B$ dont la diffusion est effective à partir du deuxième quart de ce siècle (Raynaud 1993b, 175).

Les niveaux de recharge postérieurs à l'aménagement initial du chemin n'ont fourni qu'un mobilier parcimonieux qui n'offre que des termini antérieurs à 200. Ainsi, le chemin aménagé entre 86 et 200 ne semble pas avoir fonctionné au-delà du $\mathrm{II}^{\mathrm{e}}$ siècle.

\section{LE MILIEU ENVIRONNANT}

À l'extrème fin du I ${ }^{e r} \mathrm{~s}$. et au $\mathrm{II}^{\mathrm{e}} \mathrm{s}$. les environs du cadereau ne paraissent plus tout à fait aussi ouverts qu'au cours de la période précédente. L'apport alluvial 031 qui couvre une recharge de la voie et précède la construction du mur de berge, a livré un ensemble de coquilles terrestres qui témoigne encore massivement d'un milieu ouvert et sec. Les espèces qui préfèrent l'humidité (Succinae putri, Oxyloma elegans) ne sont présentes qu'en faible quantité, comme celles qui apprécient les bosquets (Pomatias elegans Müller, Eobania vermiculata Müller). Ces dernières sont présentes à l'état de fragments, d'apex généralement corrodés ou roulés. Elles viennent peut-être d'un peu plus loin et illustreraient un secteur en amont. Dans le voisinage de la fouille, les structures agraires (fosses et fossés) de la période antérieure sont sans doute toujours utilisées, bien que leur durée de fonctionnement n'ait pas été établie avec exactitude: stratigraphiquement elles apparaissent directement sous les remblais et les niveaux labourés modernes.

L'impression de moindre ouverture du paysage vient de l'ensemble des espèces terrestres du dépôt alluvial suivant, 030. L'assemblage des espèces de milieux sec et ouvert, toujours présent, ne représente plus ici que moins du quart des individus de l'échantillon, si l'on n'y inclut pas Cecilioides acicula ". Quelques abris plus favorables sont signalés par Discus rotundatus Müller, Pomatias elegans, Ena obscura Müller et même Cochlostoma septemspirale Razoumowsky ainsi qu'une litière plus fournie par Lauria cylindracea Da Costa, et une activité souterraine marquée (25\% des terrestres sont des Cecilioides acicula Müller). La présence de Cochlicopa lubrica Müller souligne une certaine humidité de cette couche. La forte proportion de Vallonia costata Müller ne serait pas en contradiction avec cette interprétation d'un milieu plus fermé ${ }^{12}$, ou plus en mosaïque, et les 45 Carychium tridentatum Risso présents pourraient même indiquer une moins forte pression anthropique sur le site. Cette espèce, très petite $(0,8 \mathrm{~mm})$ préfère (à son échelle) les endroits tranquilles, peu fréquentés par l'homme.

Cet échantillon laisse percevoir un environnement où le couvert arboré s'est développé. Des haies ont peut-être envahi les berges du cadereau ou ont été plantées en limite des parcelles environnantes. Il est possible aussi que les terrains du secteur aient été abandonnés par les agriculteurs pendant un certain temps, et les champs laissés en friche, favorisant le développement de taillis.

\subsection{La disparition progressive du chenal}

Au cours des deux premiers siècles de notre ère, le régime alluvial du cadereau de la ZAC de la gare a progressivement diminué jusqu'à, si ce n'est s'interrompre, devenir trop faible pour occasionner le moindre transport de matériaux détritiques et donc la moindre sédimentation. Au-dessus de la dernière recharge du chemin, 029, et des dernières alluvions fines $(012$ et 011$)$, on note un changement radical dans la nature des dépôts qui colmatent le lit. Ils correspondent dorénavant à des apports latéraux : des colluvions. L'arrêt des flux liquides longitudinaux importants signe la fin de l'activité alluviale du chenal dont le lit se trouve progressivement colmaté jusqu'à ne former qu'un léger creux linéaire puis disparaitre du paysage. L'humidité reste néanmoins présente au droit du chenal où l'on perçoit toujours du ruissellement ou des poches d'eau stagnante liés à la persistance d'écoulements souterrains.

\section{LA FIN DES FLUX ALLUVIAUX}

Aux derniers dépôts alluviaux se superposent les apports $027,026,058,025=024,010,056,009,023,038,022$, $008,036,017,021=020=019,077$ et 007 . Au droit du chenal, ils exhaussent progressivement le terrain de 1,45 m. Les analyses granulométriques et micromorphologiques de 6 et 7 de ces couches se complètent pour confirmer leur origine naturelle et colluviale ( $c f$. infra annexes 4 et 5). L'uniformité des textures indique la répétition d'un même processus de mise en place des sédiments. Ceux-ci ne sont ni triés ni lités et les matériaux ont une répartition aléatoire qui illustre des dépôts effectués en vrac, sans sélection particulière. La sédimentation n'est pas continue. Entre deux phases d'accumulation, le lit, stabilisé, est marqué par le développement du couvert végétal et la formation de sols. Le degré d'évolution pédologique diffère d'un sol à l'autre,

11 Cette espèce fouisseuse peut descendre profondément selon la nature du sol; elle peut donc être, tout au moins en partie, une pollution.

12 Bien que Vallonia costata ait été mis dans le groupe 5 (stations découvertes) par Puisségur, on remarque souvent une adaptation de cette espèce à des milieux fermés et humides (Puisségur 1976). 
indiquant que ceux-ci ont connu des durées variables, plus longues après les apports 025,023 et 022 qu'au sommet de la stratigraphie $(021=020=019, c f$. annexe 5).

Si la sédimentation n'est plus que colluviale, l'eau reste néanmoins présente dans le chenal. Les espèces aquatiques, bien qu'en nette diminution, sont encore présentes: entre 4,65 et $15,16 \%$ des échantillons des couches 038 , 036 et 007 . Le niveau 017 est même exceptionnel avec une écrasante majorité d'individus aquatiques: $89,97 \%$. Il s'agit principalement d'espèces préférant les eaux temporaires qui témoignent de la persistance d'écoulements ponctuels dans le paléochenal, probablement lors des précipitations. Il est probable que lors des orages violents le cadereau ait reçu des eaux abondantes venant des berges et ait repris une activité alluviale en les drainant vers l'aval.

\section{LES INDICES DE DATATION}

Les indices de datation récoltés dans les colluvions sont extrêmement ténus et peu pertinents. Trois couches ont livré en tout 10 tessons de céramique. Les plus anciennes, 038 et 017 , contiennent des fragments de poterie antique produits au cours des $\mathrm{I}^{\text {er }}$ et $\mathrm{II}^{\mathrm{e}} \mathrm{s}$. de n.è.; ils ont pu être transportés par colluvionnement et illustrent l'érosion d'un site voisin plutôt qu'ils ne datent la sédimentation. Immédiatement au-dessus, le niveau 019 contient deux morceaux de céramiques à glaçure sur engobe et un fragment de porcelaine. Ces mobiliers ont pu être colluviés, ou correspondent aux amendements du terrain: l'étude micromorphologique révèle en effet la présence de compost dans ces niveaux ( $c f$. infra annexe 5). Ainsi les colluvions les plus anciennes sont postérieures au chemin utilisé entre la fin du Ir $s$. de n.è. et la fin du $\mathrm{II}^{\mathrm{e}} \mathrm{s}$. et les plus récentes ont été cultivées - si ce n'est formées - aux $\mathrm{XVIII}^{\mathrm{e}}$ et/ou XIX ${ }^{\mathrm{e}} \mathrm{s}$. Mille ou mille deux cents ans ont donc passé entre l'abandon du chemin et la culture attestée des terres au droit du cadereau, sans que l'on puisse établir le temps qu'il a fallu pour que celui-ci soit totalement colmaté et disparaisse du paysage.

\section{LE PAYSAGE ENVIRONNANT}

Du fait de l'imprécision de la datation des niveaux supérieurs du chenal, les témoins de l'environnement documentent de manière globale le paysage du $\mathrm{III}^{\mathrm{e}}$ au milieu XIX ${ }^{\mathrm{e}}$ siècle.

Les environs du paléochenal sont de nouveau très ouverts. Les ensembles malacologiques des couches 058,038 , 036 et 007 en témoignent avec une proportion importante de coquillages terrestres préférant les milieux secs et ensoleillés à végétation rase. La rareté des arbres est confirmée par les prélèvements polliniques qui ne livrent que quelques taxons arboréens de Pinus (pin), Abies (sapin), Acer (érable), Quercus (chêne à feuillage caduc) et Tilia (tilleul), peut-être originaires d'un milieu éloigné de plusieurs kilomètres, en particulier le pin dont les pollens sont de bons voiliers ( $c f$. annexe 7). L'écrasante majorité des pollens d'herbacées évoque principalement des lieux pâturés (Cichorioideae 32 à $48 \%$, Centaurea 4,1 à $11,6 \%$ et Poaceae 8,3 à $17,4 \%$ ) et participe aussi dans une moindre mesure de la végétation proche des habitats ou des cultures (Brassicaceae 5 à $11,4 \%$, Chenopodiaceae 4 à 16,3\%). Le secteur est néanmoins cultivé: de chanvre peut-être en partie, et de céréales surtout. Les colluvions les plus anciennes n'ont pas été remaniées après leur dépôt et ne semblent donc pas avoir fait l'objet de travaux agricoles: les cultures ont donc été pratiquées sur les abords du cadereau et ce dernier devait être couvert d'herbacées qui ont contribué à la formation des sols. En revanche, les pollens de céréales, particulièrement bien marqués dans le niveau 022 , la forte charge en matières organiques de ce niveau, associée aux remaniements postdépositionnels de la structure de cette couche, plaident pour un amendement et un labourage du sol jusqu'au droit du paléochenal. Après son colmatage, les champs ont gagné l'emprise de l'ancien cadereau qui a alors perdu son relief en creux et sa couverture végétale particulière.

Dans cet ensemble d'apports de versants, le niveau 017 se démarque. Son spectre malacologique est marqué par un impressionnant pourcentage d'individus d'espèces aquatiques et, au sein de celui-ci, par la proportion d'individus d'une seule espèce, plus de $83 \%$ de Anisus leucostomus. Une telle disproportion est généralement le signe d'un déséquilibre, d'un milieu extrême. Douze espèces aquatiques accompagnent Anisus $l$., toutes dans des proportions infimes, sauf Galba truncatula qui partage avec Anisus l. la tolérance pour des eaux temporaires ou intermittentes, et la préférence pour une végétation aquatique ou une végétation riche de berges. Cet échantillon donne l'image d'une sorte de marécage, de zone non drainée, de vasière. L'échantillon contient en masse des ostracodes (268 demicarapaces) qui prouveraient la présence d'eaux permanentes. Ce milieu humide est voisin de l'emplacement de la fouille: l'échantillon associe en effet des espèces terrestres abîmées à des espèces aquatiques généralement bien conservées. Cela suppose que les vestiges des premières ont été roulés sur de longues distances tandis que les secondes proviendraient d'une zone humide avec flaques et vases, localisée à proximité du lieu de leur dépôt.

\subsection{L'urbanisation du site}

Au-dessus des colluvions cultivés 007 et 019 , des remblais sont apportés $(006,018,005)$ qui correspondent à plusieurs installations successives de bâtiments d'habitation d'époque contemporaine sur la parcelle. Ces étapes de construction n'ont pas été traitées, de même que le remblai supérieur 001 qui correspond à la destruction de ces mêmes bâtiments dans le cadre du réaménagement du quartier de la gare. 


\section{Synthèse}

L'opération de sauvetage archéologique menée sur une petite portion du chenal de la gare a donc livré des données environnementales qui documentent l'évolution du cours d'eau et de son environnement immédiat du Ior $\mathrm{s}$. av. n.è. à nos jours. Les informations recueillies complètent avantageusement les connaissances précédemment acquises sur Nîmes, puisqu'elles concernent un secteur encore méconnu entre deux zones mieux documentées: le piémont de la Garrigue (par les études paléoenvironnementales des fouilles menées en ville depuis une quinzaine d'années), et la plaine du Vistre (par celles plus récentes réalisées dans le cadre du Plan de Protection Contre les Inondations). L'apport de l'histoire "naturelle" du quartier de la gare à la connaissance du site de Nîmes reste bien entendu insuffisant pour prétendre dresser quelque synthèse sur l'évolution particulière du milieu nîmois. Par conséquent c'est un raisonnement inverse que nous adopterons: en se rattachant à la connaissance du site et à celle, plus générale, de l'évolution du milieu pour interpréter nos découvertes.

Trois périodes ont été distinguées dans l'histoire hydrosédimentaires du cadereau de la gare. Elles se distinguent par des changements de nature des dépôts naturels ou anthropiques. Ces changements répondent à des évolutions plus générales du milieu dont les origines peuvent être climatiques ou liées à l'occupation humaine.

$\mathrm{Au} \mathrm{I}^{\mathrm{er}} \mathrm{s}$. av. n.è. jusqu'au $\mathrm{II}^{\mathrm{e}} \mathrm{s}$., le chenal est particulièrement dynamique. Le caractère torrentiel de ses dépôts témoigne de la virulence des écoulements qui le traversent et qui provoquent, au débouché du piémont des Garrigues, le débordement des eaux de crue et la possible construction d'un système de cône alluvial. Cette activité semble s'inscrire dans la période de crise hydrologique observée en moyenne vallée du Rhône et dans le Sud-Est français de l'époque augustéenne au III $\mathrm{s}$. Elle se caractérise par la montée du niveau des nappes, par la forte érosion des versants et, comme ici, par la fréquence et l'importance des crues. Son origine est peut-être climatique (Bravard et al. 1992; Berger 1996; Provansal et al. à paraître ; Berger, Brochier à paraître). Elle peut également être liée à la densification de l'occupation humaine sur les terrains du bassin versant et au développement d'activités, notamment pastorales, susceptibles de favoriser l'érosion. Cette phase de dérèglement prend fin au $\mathrm{III}^{\mathrm{e}} \mathrm{s}$. en moyenne vallée du Rhône, voire un peu après $\left(\mathrm{au} \mathrm{IV}^{\mathrm{e}} \mathrm{s}\right.$. sur les sites les plus nordiques). Lui succède alors, des Alpes à la Médi- terranée, une période de calme relatif des hydrosystèmes (Berger, Brochier à paraître) qu'illustre peut-être la diminution progressive de l'activité alluviale du cadereau de la gare après le II ${ }^{\mathrm{e}} \mathrm{s}$., puis son interruption, mal datée.

Mais ensuite, dans le quartier de la gare nîmoise, le chenal ne connait plus de phases hydrologiques très actives, alors que les études climatiques sur d'autres sites en France du sud montrent l'existence de nouveaux dérèglements entre les $\mathrm{V}^{\mathrm{e}}$ et $\mathrm{VII}^{\mathrm{e}} \mathrm{s}$., puis aux XIII $-\mathrm{XIV}^{\mathrm{e}} \mathrm{s}$. (Berger, Brochier à paraittre). Le piémont nîmois est peutêtre alors principalement drainé par d'autres cadereaux : notamment celui d'Alès et celui du Vistre de la Fontaine. La transformation du réseau hydrologique n'est pas nécessairement liée à des événements naturels. Au contraire, l'histoire de la ville de Nîmes laisse supposer que son urbanisation intense au I ${ }^{\text {er }} s$. puisse être à l'origine du détournement des flux du cadereau de la gare vers d'autres cours d'eau. En amont, la construction de l'enceinte au début de notre ère a barré la pente du site nîmois et les écoulements naturels des eaux de ruissellement ont été en partie relayés par la construction d'adductions. Certains cadereaux ont été maintenus et aménagés, notamment aux endroits où ils traversent la clôture de la ville, d'autres ont dû être abandonnés, tandis qu'au cours des décennies qui ont suivi l'édification du rempart, des collecteurs étaient bâtis pour l'évacuation des eaux domestiques usagées. Certains s'écoulaient en direction du Vistre de la Fontaine ou de ses effluents, d'autres hors les murs, vers la campagne méridionale (Monteil 1999, 357-358). Parmi ces derniers deux ont été reconnus au nord du cadereau de la ZAC de la gare. Ils sont orientés comme le cadereau luimême, mais sont situés plus à l'est (fig. $\left.4, \mathrm{n}^{\circ} 470,471\right)^{13}$. Ils ont pu se substituer au chenal de la gare pour le drainage d'une grande part des eaux du secteur.

Ainsi deux facteurs ont pu contribuer à l'interruption de l'activité alluviale du cadereau de la ZAC de la gare: des transformations climatiques qui auront conduit après le second siècle à la diminution des flux hydriques, et l'urbanisation antique de la ville de Nîmes qui a redessiné, à partir du Ier $\mathrm{s}$. de notre ère, le réseau des écoulements.

Libéré de flux importants, le cadereau a servi de voie de circulation dans un premier temps. Le chemin qui y est aménagé à partir des années 80 est perpendiculaire à l'axe formé par les rues Sainte Félicité et Sainte Perpétue (fig. $1, n^{\circ} 2$ ) qui, depuis la voie Domitienne à l'est, dessert le site antique de Sainte-Perpétue et correspond vraisemblablement à une voie antique importante au sud de la ville ${ }^{14}$. L'aménagement viaire ancien du quartier de la

13 Plus proche encore du site de la gare, le collecteur $n^{\circ} 473$ est peut-être dans le prolongement de l'égout 471 . Mais son identification est imprécise (fig. 5) (Fiches, Veyrac 1999).

14 Information de Laurent Vidal, Inrap. 
gare n'est sans doute pas une voie principale d'accès à Nîmes, la rusticité de son aménagement plaide pour un chemin de desserte locale. Il semble avoir été très vite abandonné puisqu'il ne fait plus l'objet d'entretien audelà du $\mathrm{II}^{\mathrm{e}}$ siècle.

Le chenal est alors largement abandonné. Il disparaît très progressivement sous une sédimentation colluviale qui atténue son relief en creux jusqu'à le faire disparaître du paysage. Le comblement ne suit pas un rythme homogène: il fait l'objet de plusieurs phases de sédimentation entrecoupées de périodes stables au cours desquelles des sols se sont développés. Le cadereau semble alors vierge d'occupation. Il constitue peut-être une friche linéaire dans un secteur à vocation agricole.

Le paysage environnant est en effet largement ouvert. L'approche archéologique confirme ici une occupation rurale continue qui était déjà connue par d'autres sources. Cependant les études des environnementalistes fournissent quelques indices de variation de l'intensité de l'occupation agraire. Du I ${ }^{\text {er }} s$. av. au I ${ }^{\text {er }} s$. de n.è., le paysage est très ouvert et manifestement mis en culture et en pâturage. Il se referme légèrement à la fin du $\mathrm{I}^{\mathrm{er}} \mathrm{s}$. et au $\mathrm{II}^{\mathrm{e}} \mathrm{s}$. soit parce que des taillis ou des bosquets regagnent du terrain, soit parce que la configuration du cadereau et/ou des champs se modifie avec le développement de haies en limites, soit encore parce que des arbres sont cultivés. À partir du III ${ }^{e}$ s. le paysage s'ouvre de nouveau, les témoins de pâtures et de cultures, en particulier les céréales, se multiplient. Dans les niveaux les plus récents (associés à des mobiliers des $\mathrm{XVIII}^{\mathrm{e}}$ et $\mathrm{XIX}^{\mathrm{e}} \mathrm{s}$.), alors que les cultures ont gagné l'espace du cadereau, un marécage semble se développer à proximité et en amont. Il est possible que jusqu'à sa mise en culture, le chenal ait continué de transporter quelques eaux résiduelles jusqu'au Creux des Canards, dépression humide marécageuse à quelques centaines de mètres plus au sud (fig. 1), eaux qui se seraient ensuite trouvées bloquées.

Bien que pratiquée sur une emprise retreinte et en un temps très court, la fouille échantillonnée du chenal de la gare a été fertile en données concrètes sur l'environnement suburbain nîmois. Ces résultats fructueux procèdent de la double approche archéologique et naturaliste qui s'impose de plus en plus comme incontournable, particulièrement en milieu alluvial, susceptible de documenter tout un bassin versant. L'opération archéologique a bénéficié du concours des chercheurs locaux: M. Monteil (Université) et L. Sauvage (Inrap) qui a très tôt souligné l'importance d'un tel dépôt (fût-il si peu archéologique en apparence) pour la connaissance de l'impact de l'homme et de son milieu. Qu'ils en soient tous deux sincèrement remerciés.

* INRAP.

** Association Géoarchéologie et Paysages.

*** Association de Recherche Paléoécologique en Archéologie. 


\section{BibLIOGRAPHIE}

Benoit 1981 : BENOIT (J.) - Nîmes : études sur l'urbanisme antique. Problème de méthode et résultats. Bulletin de l'École Antique de Nîmes, nouv. série, ${ }^{\circ} 16,1981$, pp. 69-90, carte grand format.

Berger 1996 : BERGER (J.-F.) - Évolution des agrosystèmes et du climat. In : FICHES (J.-L.) éd. - Le III siècle en Gaule Narbonnaise. Données régionales sur la crise de l'Empire. Actes de la table ronde du GDR 954 (Aix-en-Provence, 15-16 septembre 1995). Juan-les-Pins, A.P.D.C.A., 1996, pp. 299-332.

Berger, Brochier à paraître: BERGER (J.-F.), BROCHIER (J.-L.) Les apports de la géoarchéologie à la connaissance des paysages et des climats en moyenne vallée du Rhône. In: MAUFRAS (O.) éd. - Habitats, ensembles funéraires et paysages dans la moyenne et la basse vallée du Rhône (VII $-X V^{e}$ s.): contributions des travaux archéologiques sur le tracé du TGV-Méditerranée à l'étude des sociétés rurales médiévales. À paraitre.

Bravard et al. 1992 : BRAVARD (J.-P.), VEROT-BOURRELY (A.), SALVADOR (P-G.) - Le climat d'après les informations fournies par les enregistrements sédimentaires fluviatiles étudiés sur des sites archéologiques. Les nouvelles de l'Archéologie, 50, 1992, pp. 7-13.

Chevillot 1999 : CHEVILLOT (P.) - Approche géomorphologique de la marge nord de la plaine du Vistre. In : BREUIL (J.-Y) - Bassin Aval de basse Magaille est: habitat et réseau fossoyé du second $\hat{A} g e$ du Fer au Haut Empire. DFS de diagnostic archéologique, Montpellier, Service Régional de l'Archéologie, 1999, 110 p., ill.

Christol, Goudineau 1987 : CHRISTOL (M.), GOUDINEAU (Ch.) -

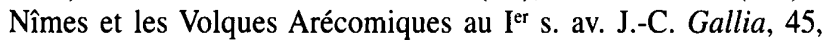
1987-1988, pp. 87-103, ill.

Fabre 2000 : FABRE (G.) - L'environnement du contact garrique-piémont à Nîmes. In: GARMY (P.), MONTEIL (M.) dir. - Le quartier des Bénédictins à Nîmes (Gard): découvertes anciennes et fouilles 1966-1992. Paris, MSH, 2000, pp. 22-27 (DAF, 81).

Fiches 1994 : FICHES (J.-L.) - Les Céramiques d'époque romaine. In: GARCIA (D.) dir. - Nouveaux résultats de l'exploration archéologique de la ville portuaire de Lattes: les îlots 2, 4-sud, 5, 7-ouest, 7-est, 8, 9 et 16 du quartier Saint-Sauveur. Lattes, ARALO, 1994, pp. 333-372 (Lattara, 7).

Fiches, Veyrac 1996 : FICHES (J.-L.), VEYRAC (A.) dir. - Carte archéologique de la Gaule: Nîmes. Paris, Fondation de la maison des sciences de l'homme, 1996, $634 \mathrm{p}$.

Germer-Durand 1874: GERMER-DURAND (É.) - Cartulaire du chapitre de l'église cathédrale Notre-Dame de Nîmes. Nîmes, Catelan, 1874, $402 \mathrm{p}$.

Manniez 2000 : MANNIEZ (Y.) - ZAC de la gare 1, Nîmes (Gard). DFS de diagnostic archéologique, Montpellier, Service Régional de l'Archéologie, 2000, 43 p., ill.
Maufras 2000 : MAUFRAS (O.) - Nîmes (Gard): ZAC de la gare III (première et deuxième tranche). Montpellier, SRA, 2000, 23 p. (DFS de diagnostic archéologique).

Mercier à paraître: MERCIER (C.) - Les villa voisines de la villa de Codols. In : POMARÈDES (X.), SAUVAGE (L.) dir. - La villa de Saint-André de Codols (Nîmes; Gard): évolution de l'habitat et de l'espace rural nîmois de l'Antiquité au Moyen Âge. À paraître.

Monteil 1999 : MONTEIL (M.) - Nimes antique et sa proche campagne: étude de topographie urbaine et périurbaine (fin $\mathrm{VI}^{e} s$. av. J.-C.-VI s. ap. J.-C.). Lattes, UMR 154 du CNRS, 1999 (MonoAMédit, 3).

Provansal et al. à paraître : PROVANSAL (M.), BERGER (J.-F.), BRAVARD (J.-P.), SALVADOR (P.-G.) - Le régime du Rhône et les mutations des environnements fluviaux du lac de Genève à la mer (à paraître dans Gallia 1998, dossier Rhône sous la dir. de $\mathrm{Ph}$. Leveau).

Puisségur 1976 : PUISSÉGUR (J.) - Mollusques continentaux quaternaires de Bourgogne. Dijon, Université de Dijon, 1976, Mém. Géol.

Py 1990 : PY (M.) - Culture, économie et société protohistoriques dans la région nîmoise. Rome, École française de Rome, 2 vol., 1990 (Coll. École française de Rome, 131).

Py 1993a: PY (M.) - Les amphores italiques. In: PY (M.) dir. Dictionnaire des céramiques antiques en Méditerranée nord-occidentale (Provence, Languedoc, Ampurdan). Lattes, ARALO, 1993, pp. 53-55 (Lattara, 6).

Py 1993b: PY (M.) - Céramique à pâte claire récente. In: PY (M.) dir. - Dictionnaire des céramiques antiques en Méditerranée nordoccidentale (Provence, Languedoc, Ampurdan). Lattes, ARALO, 1993, pp. 222-243 (Lattara, 6).

Raynaud 1993a: RAYNAUD (C.) - Céramique africaine claire A. In : PY (M.) dir. - Dictionnaire des céramiques antiques en Méditerranée nord-occidentale (Provence, Languedoc, Ampurdan). Lattes, ARALO, 1993, pp. 170-174 (Lattara, 6).

Raynaud 1993b : RAYNAUD (C.) - Céramique claire B. In : PY (M.) dir. - Dictionnaire des céramiques antiques en Méditerranée nordoccidentale (Provence, Languedoc, Ampurdan). Lattes, ARALO, 1993, pp. 175-184 (Lattara, 6).

Raynaud 1993c: RAYNAUD (C.) - Céramique commune sableuse oxydante ou réductrice du Languedoc oriental. In: PY (M.) dir. Dictionnaire des céramiques antiques en Méditerranée nord-occidentale (Provence, Languedoc, Ampurdan). Lattes, ARALO, 1993, pp. 548-553 (Lattara, 6). 